\title{
Incompressible flow assumption in hydroacoustic predictions of marine propellers
}

\author{
Savaş SEZEN ${ }^{1}$, Omer Kemal KINACI*,2 \\ ${ }^{1}$ Faculty of Naval Architecture and Maritime, Yildiz Technical University, Turkey \\ ${ }^{2}$ Faculty of Naval Architecture and Ocean Engineering, Istanbul Technical University, Turkey \\ * Corresponding author e-mail: kinacio@itu.edu.tr
}

\begin{abstract}
Incompressible flow assumption is an essential step that simplifies numerical simulations for objects inside flowing water. However, incompressibility assumption creates a conflicting situation for sound propagation as the propagation speed is given by $c_{0}=\sqrt{d p / d \rho}$ where $p$ denotes the pressure and $\rho$ denotes the density. When $d \rho=0$ is assumed to relieve simulations, speed of sound theoretically becomes infinite and therefore, induced pressures should be corrected with the acoustic analogy. This approach is called the "hybrid method" that combines hydrodynamic solver with hydroacoustic solver. Hydroacoustic solver adds compressibility effects to the incompressible hydrodynamic solver and uses hydrodynamic pressure to calculate acoustic pressure. Time step size is an important parameter to calculate acoustic pressure field in the fluid domain and an approach to determine the minimum value (for at least capturing the first blade passage frequency) is presented in this study. Another purpose of this paper is to investigate the effect of incompressibility on hydroacoustics and to analyze the necessity of utilizing the famous Ffowcs Williams-Hawkings (FWH) equation for predicting marine propeller noise; both for cavitating and non-cavitating cases. Our results are in line with other researchers; hydrodynamic pressure is sufficient to assess the hydroacoustic performance of marine propellers in the near-field due to having very low acoustic Mach numbers. Near-field results from the hydrodynamic solver are then extrapolated to the far-field by adopting ITTC distance normalization equation. However; this equation, which is actually the inverse distance law, is only valid for point noise sources in stationary flow. It is found out that eliminating FWH equation by coupling the incompressible hydrodynamic solver with ITTC distance normalization equation fails to produce satisfactory results. For cavitating cases, numerical results in this study show that implementation of $\mathrm{FWH}$ is required even in the near-field.
\end{abstract}

Keywords: acoustics; cavitation; DTMB 4119; FWH; propeller noise; tip Mach number

\section{Abbreviations}

RANS Reynolds-averaged Navier Stokes

DES Detached Eddy Simulation

DNS Direct Numerical Simulation

FWH Ffowcs-Williams and Hawkings

PPTC Potsdam Propeller Test Case

ITTC International Towing Tank Conference

BEM Boundary Element Method

SPL Sound Pressure Level

OASPL Overall Sound Pressure Level
DTMB David Taylor Model Basin

CFD Computational Fluid Dynamics

RBM Rigid Body Motion

URN Underwater Radiated Noise

VOF Volume of Fluid

SST Shear Stress Transport

BPF Blade Passage Frequency

FFT Fast Fourier Transform 


\section{Introduction}

Underwater radiated noise (URN) has been an active topic for International Maritime Organization (IMO), international associations and several classification societies to reduce the noise emission in the marine environment. Shipping activities in the oceans cause unacceptable levels of URN and damages the marine ecosystem, especially in low-frequency range (from $10 \mathrm{~Hz}$ to $1 \mathrm{kHz}$ ) (Bertschneider et al.; 2014). It was mentioned by (Andrew et al., 2002) that noise levels in the ocean was increased around $10 \mathrm{~dB}$ from 1960 to 2000 . Currently, there are studies to reduce shipping noise by $3 \mathrm{~dB}$ in 10 years and $10 \mathrm{~dB}$ in 30 years (IWC, 2009). Slow steaming is another option to reduce noise levels and it was noted by (Leaper et al., 2014) that in the last five years slow steaming reduced overall acoustic footprint by over $50 \%$. Following all these efforts to reduce ambient noise levels in the oceans, this issue was also recognized by the IMO and to reduce underwater radiated noise from commercial ships, the organization has published a non-mandatory guideline (IMO, 2014). However; today it would still be hard to say that hydro-acoustic performance of ships and propellers are investigated in detail at the design stage. Selection of propulsion system for ships only rely on propulsion efficiency (lanniello, Muscari and Di Mascio; 2013). Prediction methods of noise are mainly improved by aero-acousticians and exploited by the aerospace industry. However; with the aid of modern super-computers it is possible today to model the pressure field around the ship, which constitutes the input for hydroacoustic noise prediction. Utilization of empirical relations in the literature only provides a raw estimation of noise; further research capabilities are limited and underwater acoustic field of ships cannot be identified.

Specialist committee on hydrodynamic noise (ITTC, 2014) categorized the URN in three parts: machinery, propeller, and hydrodynamic noise. Noise induced by marine propeller is the most important one, especially in the presence of cavitation. Marine propeller noise can be predicted using experimental methods in cavitation tunnels or by numerical methods and empirical estimations. In numerical calculations, acoustic analogies are used together with the hydrodynamic solver as a hybrid approach. In the past, several studies were conducted to investigate the propeller hydroacoustic performance through numerical tools. Salvatore and lanniello, (2003) investigated acoustic pressure field induced by cavitating marine propellers. A potential based BEM solver was coupled with acoustic analogy method based on FWH approach. Numerical hydroacoustic pressures obtained by FWH were compared with the classical Bernoulli equation approach. Effects of cavitation on propeller hydroacoustic performance were investigated. Seol, Suh and Lee (2005) examined the propeller radiated noise in uniform and non-uniform flow under non-cavitating and blade sheet cavitation conditions. Hydrodynamic part was computed by using a potential based panel code, whereas noise signature of the propeller was estimated by coupling the code with acoustic analogy. Contributions made by thickness and noise terms were investigated for different hydrophone locations. It was found that dipole noise term dominates the noise directivity pattern, especially under non-cavitating conditions. Ianniello, Muscari and Di Mascio (2011) used the acoustic analogy to predict the noise induced by marine propeller. Hydrodynamic simulations were conducted with RANS (Reynoldsaveraged Navier Stokes) equations and permeable FWH equation was used to predict the noise level. These simulations were conducted both in open water and behind the ship conditions. Contributions of linear and non-linear terms of the FWH equation were investigated. Hallander et al., (2012) focused on the propeller noise behind the ship wake. Different viscous and potential hydrodynamic solvers were used together with FWH acoustic analogy to predict the cavitating propeller noise. Noise predictions due to sheet and tip vortex cavitations were done in both near and far field conditions. It was mentioned that RANS coupled FWH equation gives acceptable results in near field when compared with sea trial data. (Ianniello, Muscari and Di Mascio; 2013) studied on the hydroacoustic characteristics of well known E779A model propeller in uniform flow conditions. In these simulations, 
RANS with permeable FWH equation were used to investigate the functionality of the hydrodynamic pressure for noise estimation with dynamic overset grid. Effects of linear and non-linear terms on propeller noise signature were examined at different receiver positions. Lloyd, Rijpkema and Wijngaarden (2015) investigated the propeller hydroacoustic performance using RANS with FWH acoustic analogy in open water condition. Hydrodynamic and hydroacoustic simulations were conducted both for steady and unsteady conditions. Artificial time step was defined to extract the time series of acoustic pressures. Pressures which are obtained directly by solving hydrodynamic solver and RANS with FWH equation were compared to each other. lanniello and De Bernardis, (2015) studied on the potential of acoustic analogy in the prediction of propeller hydroacoustic performance. In these simulations, incompressible hydrodynamic code with an acoustic analogy based on FWH equation was used. RANS and DES models were used to compare the vortex field for noise estimation. It was said that the non-linear quadrupole terms should not be neglected for noise estimation regardless of propeller rotational speed. Lidtke, Humphrey and Turnock (2016) investigated the hydrodynamic and hydroacoustic performances of PPTC test case and NACA0009 sections. FWH acoustic analogy was used to predict the propeller tonal blade passage noise. Besides, cavitating flow around the hydrofoil was also solved by using LES. Numerical results showed that low-frequency cavitation noise can be predicted by proposed numerical approach and acoustic analogy. Testa, lanniello and Salvatore (2017) examined tonal noise induced by marine propeller using a novel hydroacoustic formulation in the presence of sheet cavitation under non-homogenous flow conditions. Advantages and disadvantages of the method was tested for different loading conditions. Porcacchia et al., (2018) investigated noncavitating marine propeller noise under non-uniform flow conditions. Permeable FWH acoustic analogy was used for solving with BEM to predict the noise level induced by an inclined marine propeller. Velocity and pressure field for permeable surface were obtained by DES. In near field, acoustic pressures and hydrodynamic pressures were compared to each other.

All studies given above investigated the propeller hydroacoustic performance under uniform and nonuniform flow conditions. These numerical noise measurements of marine propellers were generally carried out by adopting a hybrid method and superimposing hydrodynamic and hydroacoustic solvers. In this approach, hydrodynamic pressure obtained from incompressible momentum equations (RANSE) are corrected to add compressibility effects and obtain the acoustic pressure. Present study deals with prediction of propeller radiated noise by using the same method under uniform (open water) conditions. Next section presents mathematical background of the study including incompressible hydrodynamic solver equations and $\mathrm{FWH}$. Equations regarding hydrodynamic and hydroacoustic propeller performance are also included in the same section. Remaining part of this study is organized as follows: Section 3 presents numerical implementation of mathematical background by explaining the grid structure, numerical algorithms and selection of time step size. Verification and validation study is conducted and presented in Section 4. Results both for noncavitating and cavitating cases are provided in Section 5. Section 6 is devoted to discussion of main points of this study. Paper is finalized with conclusions presented in Section 7.

\section{Mathematical background}

Mathematical background in this study can be categorized in three sections. First subsection introduces fundamental equations of incompressible computational fluid dynamics. Second subsection presents Ffowcs Williams-Hawkings equation that allows prediction of far-field noise. This method is applicable when hydroacoustic solver is coupled with incompressible hydrodynamic solver. Third section briefly lists the equations related to hydrodynamic and hydroacoustic properties of a propeller that are used in this study. 


\subsection{Incompressible hydrodynamic solver equations}

Hydrodynamic solver utilizes incompressible form of continuity and momentum equations. Four equations in a three-dimensional environment (one continuity and three momentum equations) constitute the famous Navier-Stokes equations. Incompressible form of continuity equation is given as:

$$
\nabla \cdot \vec{u}=0
$$

Incompressible form of the turbulent conservation equation is given as:

$$
\rho\left(\frac{\partial \vec{u}}{\partial t}+[\vec{u} \cdot \nabla] \vec{u}\right)=-\nabla p+\mu\left(\nabla^{2} \vec{u}\right)+\rho \vec{g}-\nabla \cdot\left(\overline{u^{\prime} \overrightarrow{u^{\prime}}}\right)
$$

Last term in the momentum equation represents the turbulent fluctuations of flow variables. Utilising suitable turbulence model will bring extra equations where additional unknown variables are introduced as the turbulent fluctuations in equation (2).

Incompressible flow equations presented in this section will return pressure values assuming that speed of sound is infinite. For more accurate noise predictions, compressible forms of conservation equations must be solved. However; computationally, this is very expensive for far-field noise estimations. Conventional method will introduce compressibility into flow by using acoustic analogy which is presented in the next section.

\subsection{Formulation and solution of Ffowcs Williams-Hawkings equation}

Noise predictions were made by homogeneous acoustic wave equation until inhomogeneous form of this equation was presented by (Lighthill; 1952). Acoustic analogy presented by Lighthill was improved by Ffowcs Williams and Hawkings (1969) who rearranged the mass and momentum equations to include moving bodies for aerodynamically generated sound.

Consider a volume $V$ that consists of two regions enclosed by $\Sigma$ as in Figure 1 . Volume consists of two regions and one of these regions (R1) is moving with a velocity $\vec{v}$ and overrunning on the other region (R2). Continuity equation in this case becomes;

$$
\frac{\partial \bar{\rho}}{\partial t}+\nabla \cdot(\overline{\rho \vec{u}})=\rho_{0} \vec{v} \delta(f) \Delta f
$$

In this equation, overbar denotes mean value of the variable, $\rho_{0}$ is the density of undisturbed medium and $\delta(f)$ is the delta function which is zero everywhere except $f=0$; which defines the surface of region 1 . Similarly, momentum equation is;

$$
\frac{\partial \overline{\rho \vec{u}}}{\partial t}+\nabla \cdot(\overline{\rho \vec{u}}+\bar{P})=P \delta(f) \Delta f
$$

Time derivative of the continuity equation and the gradient of momentum equation should be taken to eliminate $\overline{\rho \vec{u}}$. Subtracting momentum equation from continuity equation in this case presents Ffowcs Williams-Hawkings equation, which is given as;

$$
\overline{\mathbb{D}}^{2} p^{\prime}=\frac{\partial}{\partial t}\left[\rho_{0} \vec{v} \delta(f) \Delta f\right]-\nabla \cdot[P \delta(f) \Delta f]+\nabla \cdot \nabla \cdot[T H(f)]
$$

Here; $\overline{\mathbb{D}}$ defines the $\mathrm{D}^{\prime}$ Alembert operator, $P$ is the compressive stress tensor, $T$ is the Lighthill stress tensor and $H(f)$ is the Heaviside delta function. $p^{\prime}$ denotes the acoustic pressure and defined by $p^{\prime}=$ 
$p-p_{0}=c^{2}\left(\rho-\rho_{0}\right)$. For an extensive explanation on the derivation of this equation and in-depth definitions of the variables, readers can refer to (Ffowcs Williams and Hawkings; 1969).

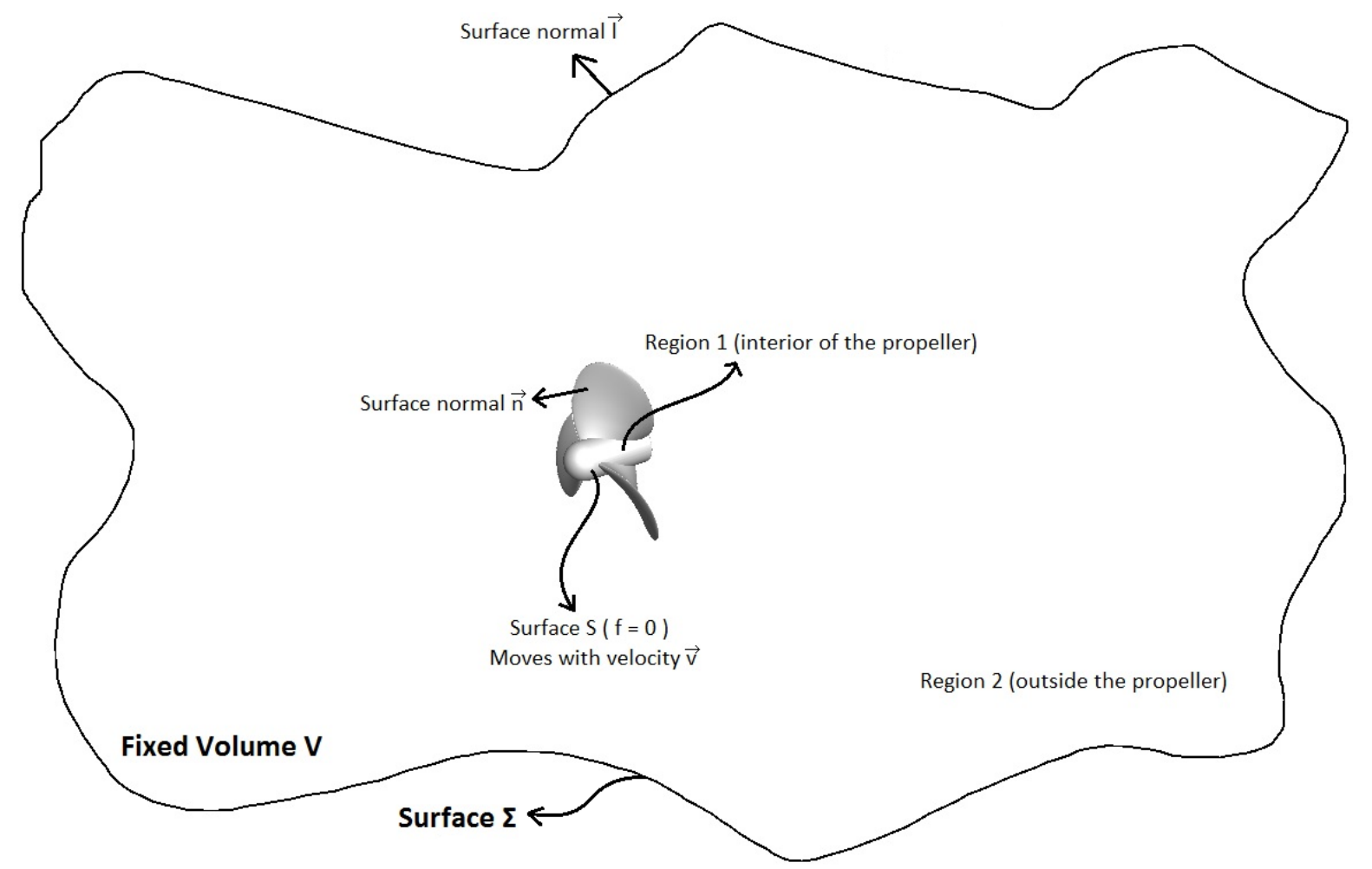

Figure 1. Problem definition of inhomogeneous acoustic wave equation for moving bodies.

Farassat proposed a solution of this equation without considering the quadrupole terms (which is the third term on the right hand side). By setting the first and second terms at the right hand side, as the monopole and dipole sources respectively;

$$
\begin{aligned}
\overline{\mathbb{D}}^{2} p_{T}^{\prime} & =\frac{\partial}{\partial t}\left[\rho_{0} \vec{v} \delta(f) \Delta f\right] \\
\overline{\mathbb{D}}^{2} p_{L}^{\prime} & =-\nabla \cdot[P \delta(f) \Delta f]
\end{aligned}
$$

he proposed numerical solutions for these two equations by using Green Function:

$$
\begin{gathered}
4 \pi p_{T}^{\prime}(x, t)=\int_{f=0}\left[\frac{\rho_{0} \dot{v}_{n}}{r\left(1-M_{r}\right)^{2}}+\frac{\rho_{0} v_{n} \widehat{r}_{l} \dot{M}_{l}}{r\left(1-M_{r}\right)^{3}}\right]_{r e t} d S \\
\quad+\int_{f=0}\left[\frac{\rho_{0} c v_{n}\left(M_{r}-M^{2}\right)}{r^{2}\left(1-M_{r}\right)^{3}}\right]_{r e t} d S \\
4 \pi p_{L}^{\prime}(x, t)=\int_{f=0}\left[\frac{\dot{P} \cos \theta}{c r\left(1-M_{r}\right)^{2}}+\frac{\widehat{r}_{l} \dot{M}_{l} P \cos \theta}{c r\left(1-M_{r}\right)^{3}}\right]_{r e t} d S \\
+\int_{f=0}\left[\frac{P\left(\cos \theta-M_{i} n_{i}\right)}{r^{2}\left(1-M_{r}\right)^{2}}+\frac{\left(M_{r}-M^{2}\right) P \cos \theta}{r^{2}\left(1-M_{r}\right)^{3}}\right]_{r e t} d S
\end{gathered}
$$

In these equations, $p_{T}^{\prime}$ represents the thickness noise while $p_{L}^{\prime}$ represents the loading noise. Monopole noise term arises by displacement of the fluid as the blade rotates, whereas dipole noise term occurs from unsteady motions of force distribution on the propeller blade surface. These two equations add up to form the impermeable formulation of Farassat; which is also known as 'Formulation $1 A^{\prime}$ ' (Farassat, 2007): 


$$
p^{\prime}(x, t)=p_{T}^{\prime}(x, t)+p_{L}^{\prime}(x, t)
$$

It is stated in various papers that linear terms dominate nonlinear terms in the propeller plane (Ianniello et al., 2013; Lloyd et al., 2015). Therefore, implementation of impermeable formulation is considered to be fair since hydrophones in this study are located in the radial direction and near field (see Figure 6).

Collapse of cavity bubbles can significantly alter pressures for very short durations (Brennen, 2008). This effect, also called as bursting, was left out of the problem. Readers interested in cavity collapse are referred to the study of (Salvatore et al., 2009) where have presented a FW-H/TVM (transpiration velocity model) to account for deformations due to growth and collapse of the cavity. Their method uses the porous $\mathrm{FW}-\mathrm{H}$ and a cavitation transpiration velocity equation, additionally.

\subsection{Equations regarding hydrodynamic and hydroacoustic properties of a propeller}

Hydrodynamic performance of a propeller is evaluated in open-water tests. Thrust $T$ and torque $Q$ generated by the propeller are measured for different advance ratios $J$ of the propeller and these variables are non-dimensionalized to give thrust and torque coefficients; $K_{T}$ and $K_{Q}$ :

$$
\begin{aligned}
& K_{T}=\frac{T}{\rho n^{2} D^{4}} \\
& K_{Q}=\frac{Q}{\rho n^{2} D^{5}}
\end{aligned}
$$

In these equations; $\rho$ is the water density $\left(\mathrm{kg} / \mathrm{m}^{3}\right), n$ is the number of revolutions per second $(r p s)$ and $D$ is the diameter of the propeller $(m)$. For a marine propeller, open-water efficiency $\eta_{0}$ is calculated by the ratio of thrust over torque;

$$
\eta_{0}=\frac{J}{2 \pi} \frac{K_{T}}{K_{Q}}
$$

Advance ratio is $J$ defined by:

$$
J=\frac{V_{A}}{n D}
$$

$V_{A}(m / s)$ is the incoming uniform flow velocity received by the propeller. Non-dimensional equation expressing cavitation is called the cavitation number and denoted by $\sigma$ which is given by;

$$
\sigma=\frac{P_{0}-P_{V}}{\frac{1}{2} \rho(n D)^{2}}
$$

Free surface effects are not included in this study; therefore, hydrostatic pressure is not included in the cavitation equation. $P_{V}$ is the vapor pressure of water $(\mathrm{Pa})$ at $17^{\circ} \mathrm{C}$ and $P_{0}$ is the static pressure $(\mathrm{Pa})$ given by:

$$
P_{0}=P_{a t m}+\rho g h
$$

Here; $P_{a t m}$ is the atmospheric pressure $(P a), g$ is the gravitational acceleration $(m / s)$ and $h$ is the submergence depth $(m)$ taken as $h=5 m$ in this study. Sound waves move in elastic media and the compressibility of the fluid is important for sound propagation. Rotation speed of the propeller is important for hydroacoustic analysis and this is non-dimensionalized by the tip Mach number $M a_{\text {tip }}$; 


$$
M a_{t i p}=\frac{n \pi D}{c}
$$

where $c$ is the speed of sound in water. Blade passage frequency $B P F$ of a propeller is a decisive factor affecting the pressure fluctuations in the fluid. It also significantly affects the sound pressure levels in the frequency domain. $B P F$ is given by:

$$
B P F=k_{f} n Z
$$

$k_{f}$ is the harmonic number (and equal to 1 for the $1^{\text {st }} B P F$ ) and $Z$ denotes the number of blades that the propeller has.

\section{Numerical implementation}

This section presents the grid system used to solve the flow around the propeller and how numerical algorithms were selected. A special subsection is devoted only to time step size as it is considered to be highly important for correct noise predictions.

\subsection{Discretization of the fluid domain}

Computational domain and domain dimensions representing the open water test conditions are depicted in Figure 2. Here, the right side of the computational domain was defined as velocity inlet while the left side was pressure outlet. Remaining surfaces were defined as symmetry plane. Propeller blades and shaft were identified as no-slip wall; imposing the zero normal and tangential velocities and satisfying the kinematic boundary condition on the propeller blades. Inside the larger static fluid domain, a rotating region surrounding the propeller was created. This block of fluid (along with its mesh) was moving together with the propeller while the rest of the domain was stationary.
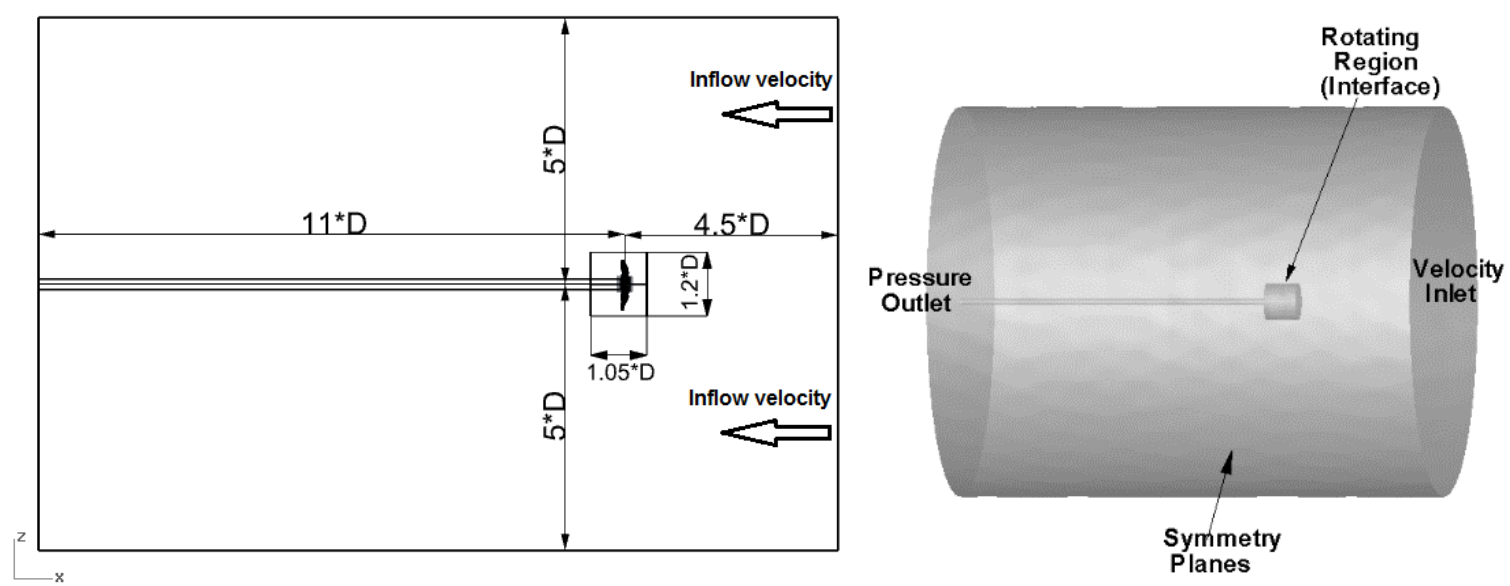

Figure 2. A summary of the computational domain for numerical simulations.

Computational domain was majorly discretized by using hexahedral elements. There were few tetrahedral elements in the domain as well; especially near the walls where the geometry is most complex. Local mesh refinements were implemented specifically around the near field region of the propeller for better approximation of the pressure field. Unstructured mesh around the propeller is presented in Figure 3. 


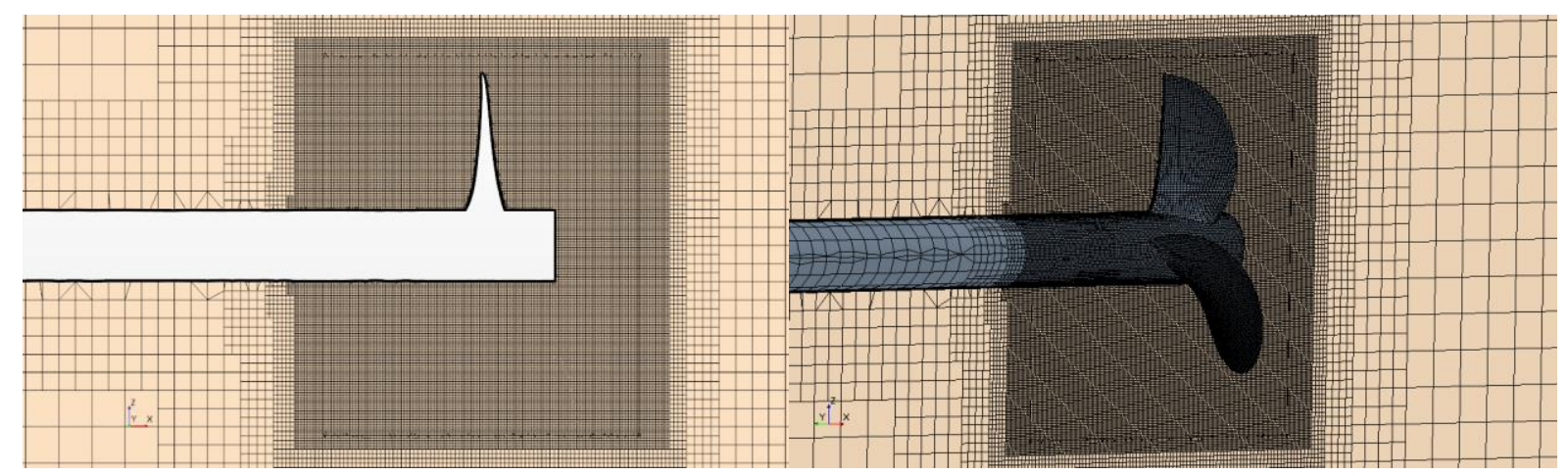

Figure 3. The unstructured mesh around the propeller.

\subsection{Numerical algorithms}

Governing equations of fluid motion were solved by using a commercial computational fluid dynamics (CFD) software Star CCM+. Cavitating and non-cavitating flow around the model propeller were solved by using unsteady RANSE (Reynolds-averaged Navier Stokes Equations) approach implementing SST $k-\omega$ turbulence model. Pressure-velocity coupling was handled by adopting SIMPLE algorithm to solve for the pressure field. Segregated flow model was implemented by using a first order implicit unsteady numerical scheme. Cavitation was modeled by using Volume of Fluid Approach (VOF) with Schnerr-Sauer cavitation model based on Rayleigh-Plesset equation. A hybrid method, which couples unsteady RANSE with FWH equations, were used to predict the propeller radiated noise. In the coupled approach, RANSE and FWH are communicating through pressures. RANSE-based results of pressure induced in the flow due to rotating propeller are given as an input to $\mathrm{FWH}$, which transfers results to the far-field. Although the propeller might be cavitating, effect of quadrupole sources is not considered. It is known that, quadrupole sources have minor effect in the near-field and linear terms are dominant in the vicinity of propeller plane (Ianniello et al., 2013). All hydrophones in this study are in the propeller plane and very close to the propeller.

Propeller rotation was represented by Rigid Body Motion or Sliding Mesh (RBM) technique. Rigid Body Motion (Sliding Mesh/RBM) method is the rotating mesh with sliding interface which is considered as the most accurate way to solve unsteady problems. RBM method involves accurate time dependent simulations where transient features of the flow are most significant. At each time step, unsteady governing equations are solved for each cell and the communication with the interface region is made by calculation of the fluxes at the boundaries of the interface. Both methods give similar results in terms of propeller hydrodynamic characteristics. However, several studies such as (Kellett, Turan and Incecik; 2013), (Noughabi, Bayati and Tadjfar; 2017), (Moussa; 2014) and (Hallander et al., 2012) state that RBM method generates better results for prediction of propeller noise under cavitating and noncavitating conditions.

\subsection{Selection of time step size}

Sound pressure levels (SPL) in frequency domain for a specific position depends on correct approximation of pressures at those points. Existence of a rotating propeller creates oscillations in pressure and a good estimation of pressure fluctuations heavily relies on the selection of time step size. In this section, how time step size is selected in numerical simulations for better sound pressure levels estimation is explained.

Let us assume that we have a propeller that has only one blade $Z=1$ which rotates at $n=1 \mathrm{rps}$. At an arbitrary point in the flow, we want to calculate the pressure in time. A schematic view is provided in Figure 4 (left). Pressure fluctuations usually look like a sine curve given in Figure 4 (right) and for (at 
least) capturing the $1^{\text {st }} \mathrm{BPF}$, it is considered that this sine curve should be represented by at least 9 points. A better approximation of the curve can be achieved by selecting more points representing the curve and this would also yield sound pressure levels at higher frequencies. However; trying to approximate the curve with less than 9 points will generate a straight line between two consecutive points.

Rotation of the propeller is $n$ and the blade passage frequency is $B P F=k_{f} n Z$ as given in equation (18). Let $k$ denote the number of representation points for the pressure curve; therefore, it may be stated that the time step size should be;

$$
\Delta t \leq \frac{1}{k \cdot B P F}=\frac{1}{k \cdot k_{f} n Z}
$$

If we go back to our example given in Figure 4 , number of blades was $Z=1$ and the rotation rate was $n=1$ rps. Number of representation points was $k=9$. In this case, time step size should be $\Delta t \leq$ $1 / 9 \mathrm{~s}$. Fast Fourier Transform (FFT) is needed to convert the data from time domain to frequency domain. While doing FFT, number of data points should be power of 2; therefore, for 1 second of hydroacoustic simulation time step size should be,

$$
\Delta t=\frac{1}{2^{m}}
$$



Figure 4. Schematic representation of a propeller with one blade (left). A typical pressure graph of point $\mathrm{P}$ due to propeller rotation (right).

Substituting equation (20) into equation (19) and rearranging the equation,

$$
m \geq \log _{2} k_{f} k Z n
$$

where $m$ is an integer. Applying the values of our example $k_{f}=1, k=9, n=1$ rps and $Z=1$ into this equation; we find $m=4$, and the time step size becomes $\Delta t=1 / 16 \mathrm{~s}$. It should be noted here again that with this selection of time step size, only the $1^{\text {st }}$ BPF can be captured. If higher harmonics are required, than the value of $m$ will be greater which will lead to a smaller time step size.

As another example, a propeller with $Z=4$ number of blades and a rotation rate of $n=10 \mathrm{rps}$ should have a time step size of at least $\Delta t \leq 1 / 360 s$ to build the pressure versus time curve for correct sound 
pressure level calculations. Keeping in mind FFT process at the end, we calculate $m$ from equation (21) and find $m=9$. In this case, time step size should be $\Delta t=1 / 512 \mathrm{~s}$ using equation (20).

This time step size selection process is adopted to prevent the commercial CFD codes to extrapolate data points while doing FFT when there is missing data. For example, if the time step size is selected as $\Delta t=1 / 500 \mathrm{~s}$, then the missing 12 data points is compensated by extrapolation from the existing results. It is considered that the method explained in this work for selecting time step size would better reflect the true nature of hydroacoustic simulations, especially for the $1^{\text {st }}$ BPF.

\section{Verification and validation of numerical simulations}

Verification and validation study was conducted to identify the uncertainty level of numerical simulations. The study is based on the methodology of (Stern et al.; 2001). Due to the absence of hydroacoustic experimental results, hydrodynamic results obtained from open-water propeller tests were used to assess uncertainty. Thrust coefficient was taken as the integral variable for the openwater propeller condition at $J=0.5$. After the verification study, a validation study was carried out by using the experimental results found in (Jessup; 1989).

Table 1. Simulation results with different grids.

\begin{tabular}{|c|c|c|c|}
\hline & Grid 3 & Grid 2 & Grid 1 \\
\hline No. of elem. & $2.4 M$ & $6.8 M$ & $19 M$ \\
\hline$S_{U}$ & 0.3189 & 0.3125 & 0.3033 \\
\hline$S_{L}$ & 0.3182 & 0.3122 & 0.3019 \\
\hline$S_{G}$ & 0.3186 & 0.3124 & 0.3026 \\
\hline$U_{I}$ & $3.5 * 10^{-4}$ & $1.5 * 10^{-4}$ & $7 * 10^{-4}$ \\
\hline $100 * U_{I} / S_{G}$ & 0.11 & 0.05 & 0.23 \\
\hline
\end{tabular}

Table 2. Simulation results with different time steps.

\begin{tabular}{|c|c|c|c|}
\hline & Time Step 3 & Time Step 2 & Time Step1 \\
\hline$\Delta t$ & $1 / 256$ & $1 / 512$ & $1 / 1024$ \\
\hline$S_{U}$ & 0.3100 & 0.3125 & 0.3169 \\
\hline$S_{L}$ & 0.3090 & 0.3122 & 0.3159 \\
\hline$S_{G}$ & 0.3095 & 0.3124 & 0.3164 \\
\hline$U_{I}$ & $0.5 * 10^{-4}$ & $1.5 * 10^{-4}$ & $5 * 10^{-4}$ \\
\hline $100 * U_{I} / S_{G}$ & 0.16 & 0.05 & 0.16 \\
\hline
\end{tabular}

Total numerical simulation uncertainty $U_{S N}$ is considered to have iterative uncertainty $U_{I}$, grid uncertainty $U_{G}$ and the time step uncertainty $U_{T}$;

$$
U_{S N}=\sqrt{{U_{I}}^{2}+U_{G}{ }^{2}+U_{T}^{2}}
$$


Three different grids and three different time steps were used to calculate $U_{G}$ and $U_{T}$ respectively. Element numbers were $2.4 \mathrm{M}, 6.8 \mathrm{M}$ and $19 \mathrm{M}$. Time step sizes were $1 / 256 \mathrm{~s}, 1 / 512 \mathrm{~s}$ and $1 / 1024 \mathrm{~s}$. The results of the simulations are given in Table 1 for $U_{G}$ and Table 2 for $U_{T}$.

In Table 1 and Table 2, $S_{U}$ and $S_{L}$ refer to the upper and lower values obtained from the last period of the thrust coefficient oscillation after convergence is determined. Last rows in these tables reveal the percentage of uncertainty with respect to obtained thrust coefficient (i.e. for grid 2, iterative uncertainty is $U_{I} \approx 0.05 \% S_{g}$ ). It may be said that the iterative uncertainties are low compared to grid and time step uncertainties.

Grid refinement ratio was taken as $r_{G}=\sqrt{2}$ and the time steps were increased with a refinement ratio of 2 . Simulation with different grids reached an oscillatory convergence $\left(R_{G}>1\right)$ while different time steps reached a monotonic convergence $\left(R_{G}<1\right)$. Values of uncertainty are given in Table 3 for grid 2 (6.8M elements) and time step $2(\Delta t=1 / 512)$.

Table 3. Uncertainty percentages with respect to the simulation results.

\begin{tabular}{|c|c|c|}
\hline & Value & $\% S$ \\
\hline$U_{I}$ & $1.5 * 10^{-4}$ & 0.05 \\
\hline$U_{G}$ & $1.880 * 10^{-2}$ & 6.02 \\
\hline$U_{T}$ & $1.932 * 10^{-2}$ & 6.19 \\
\hline$U_{S N}$ & $2.696 * 10^{-2}$ & 8.63 \\
\hline
\end{tabular}

Experimental thrust coefficient from the reference study (Jessup; 1989) is $D_{E}=0.29$ while the simulation results in this study suggest $S=0.3124$. Error is given by $E=D_{E}-S$ and it is calculated as $E=-2.24 * 10^{-2}$. The reference study does not provide information about experimental uncertainty; therefore, validation uncertainty is taken as $U_{V}=U_{S N} \ln$ this case $|E|<U_{V}$ and the simulation results are validated. Using $6.8 \mathrm{M}$ elements and a time step size of $\Delta t=1 / 512 \mathrm{~s}$, the openwater numerical simulations were carried out for the other advance coefficients as well. Computational versus experimental open-water propeller performance is given in Figure 5.

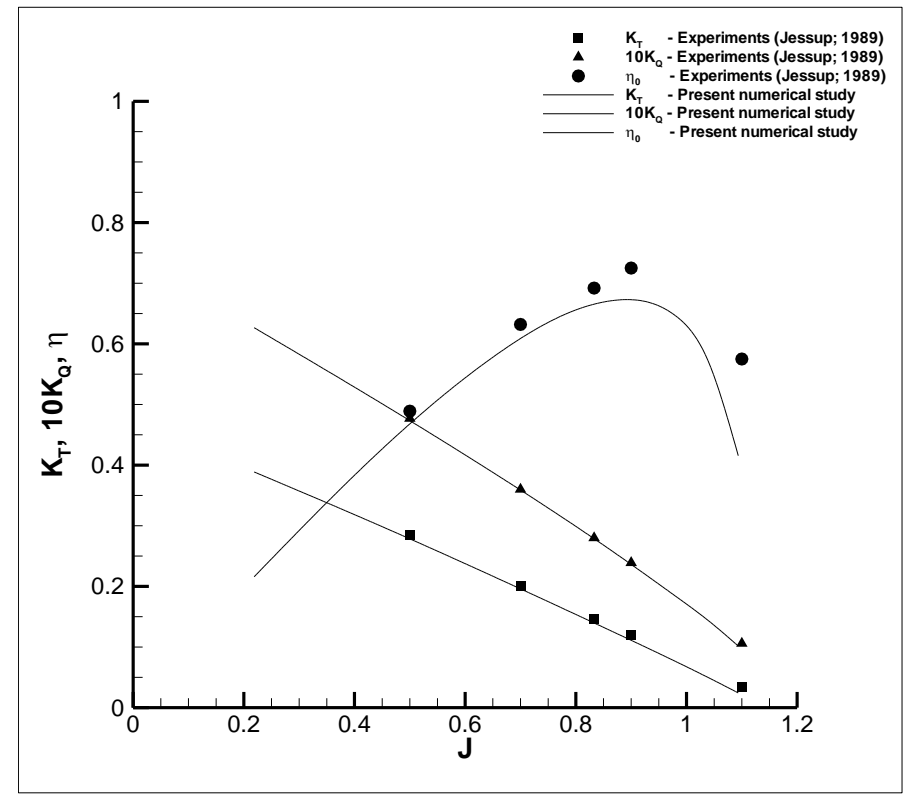

Figure 5. Open-water propeller performance of DTMB 4119 propeller. 
Error margin and the level of uncertainty in numerical simulations would have been smaller if a steady solver was used in this study. In terms of the hydrodynamic performance of the propeller, it is noted by (Viitanen and Siikonen, 2017) that quasi-steady simulations are accurate enough. They state that steady cavitation patterns can be observed with this approach. However, the focus of this study is not only to track the hydrodynamic but also the hydroacoustic properties of DTMB 4119 propeller. Therefore; time step size uncertainty was also included in calculations, which is very crucial for numerical noise estimation.

\section{Results}

After maintaining the uncertainty level of computational simulations, numerical results were derived for non-cavitating and cavitating cases. Simulation conditions are similar for both cases to directly compare the results. The only difference between the two cases is the lower static pressure imposed on the latter to lower the cavitation number and create a sheet cavitation at the back side of blades.

\subsection{Non-cavitating case}

Non-cavitating case of the open-water DTMB 4119 propeller was investigated in this section. The study of Seol et al. (2002) was one of the first that calculated the hydroacoustic properties of this propeller but at a very low rotation rate of $n=2 r p s$ and in non-uniform flow. Later, Gungor and Ozdemir (2018) used results of Seol et al. (2002) and validated their numerical tool. In this section, the original DTMB 4119 propeller with a diameter of $D=0.3048 \mathrm{~m}$ rotates at $n=10 \mathrm{rps}$ in uniform flow with velocity $V=1.524 \mathrm{~m} / \mathrm{s}$. The propeller is totally submerged in water $(h=5 \mathrm{~m})$ and is independent of free surface effects. Atmospheric pressure is set as $P_{a t m}=101.325 \mathrm{kPa}$ returning a high cavitation number $\sigma=31.92$ and therefore cavitation is not expected. Simulation conditions and geometric properties for the non-cavitating open-water DTMB 4119 propeller are given in Table 4.

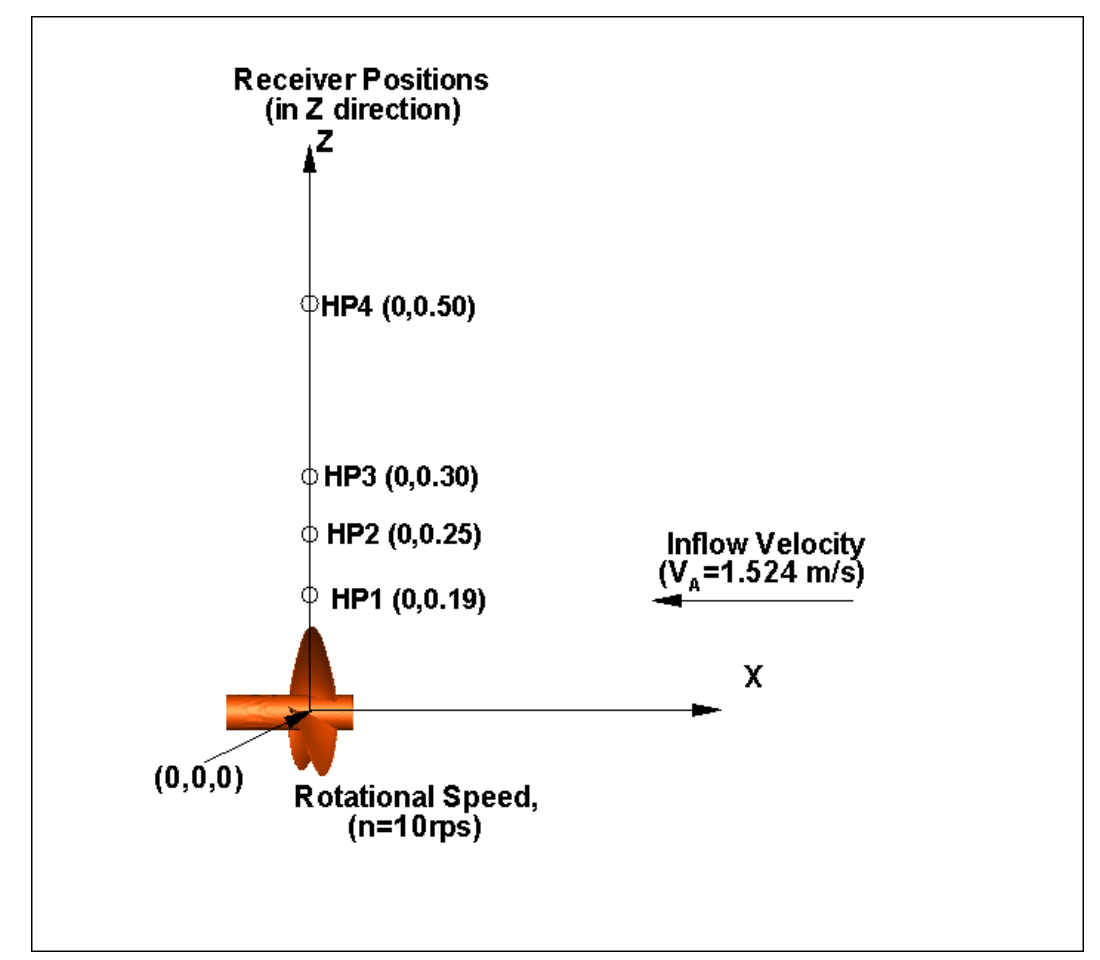

Figure 6. Positions of hydrophones located in the radial direction. Figure is illustrative and not to scale. 
Four hydrophones are positioned in the propeller disk plane. No points in the wake of the propeller are selected due to high effect of turbulence on noise for this region. Resolving turbulence in the wake region of a propeller requires advanced models which is out of the scope of this study (Lloyd, Rijpkema and Wijngaarden, 2015). Hydrophones (sometimes abbreviated by HP in this study) are located in the radial direction as shown in Figure 6.

Pressure data obtained from numerical simulations are given in Figure 7 for hydrophones 1 and 2 and Figure 8 for hydrophones 3 and 4. Pressure fluctuations in time domain are obtained from the solution of Navier-Stokes equations for "incompressible solver" results while the results denoted by "FWH" are obtained by coupling the hydrodynamic solver with the hydroacoustic solver that numerically solves Ffowcs Williams-Hawkings equation.

Table 4. Simulation conditions and geometric properties of the non-cavitating DTMB 4119 propeller.

\begin{tabular}{|c|c|c|c|c|c|c|c|}
\hline $\mathrm{D}(\mathrm{m})$ & $\mathrm{n}(\mathrm{rps})$ & $\mathrm{V}(\mathrm{m} / \mathrm{s})$ & $\mathrm{P}_{0}(\mathrm{~Pa})$ & $\mathrm{J}(-)$ & $\sigma(-)$ & $\mathrm{Ma}_{\text {tip }}(-)$ & $1^{\text {st }} \mathrm{BPF}(\mathrm{Hz})$ \\
\hline 0.3048 & 10 & 1.524 & 150255 & 0.5 & 31.92 & $6.4^{*} 10^{-3}$ & 30 \\
\hline
\end{tabular}

Pressure fluctuations obtained from hydrodynamic solver for one propeller rotation at hydrophones 1 and 2 nearly coincide with the results obtained by implementation of FWH equation. However, as the hydrophone distance from the propeller becomes larger, incompressibility assumption starts to become visible in results. Pressure data given for hydrophone 3 shows a slight change in amplitude and frequency while the deviation between results are larger for hydrophone 4 . There are several research papers that address this issue of RANSE. As the distance to the noise source becomes larger, numerical diffusion leads to accumulation of error and reliability of RANSE gets lost (Lloyd, Rijpkema and Wijngaarden; 2015). A similar case is also mentioned in (lanniello, Muscari and Di Mascio; 2013). Based on Figure 7 and Figure 8, pressure data obtained only from incompressible hydrodynamic solver is sufficient to assess propeller noise in the near-field, while utilization of hydroacoustic solver is required to calculate the noise in the far-field.

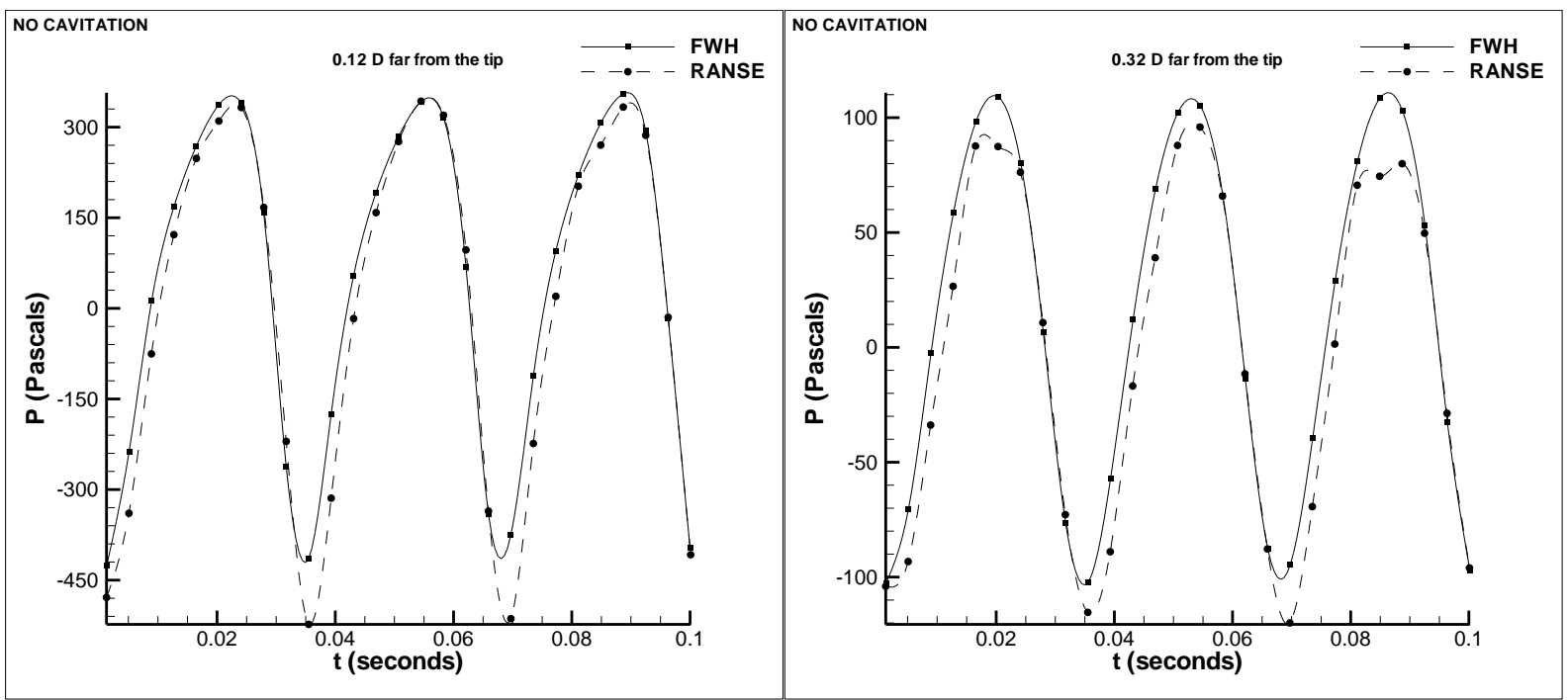

Figure 7. Pressure fluctuations induced by one propeller rotation at hydrophones 1 (left) and 2 (right) for non-cavitating case. 


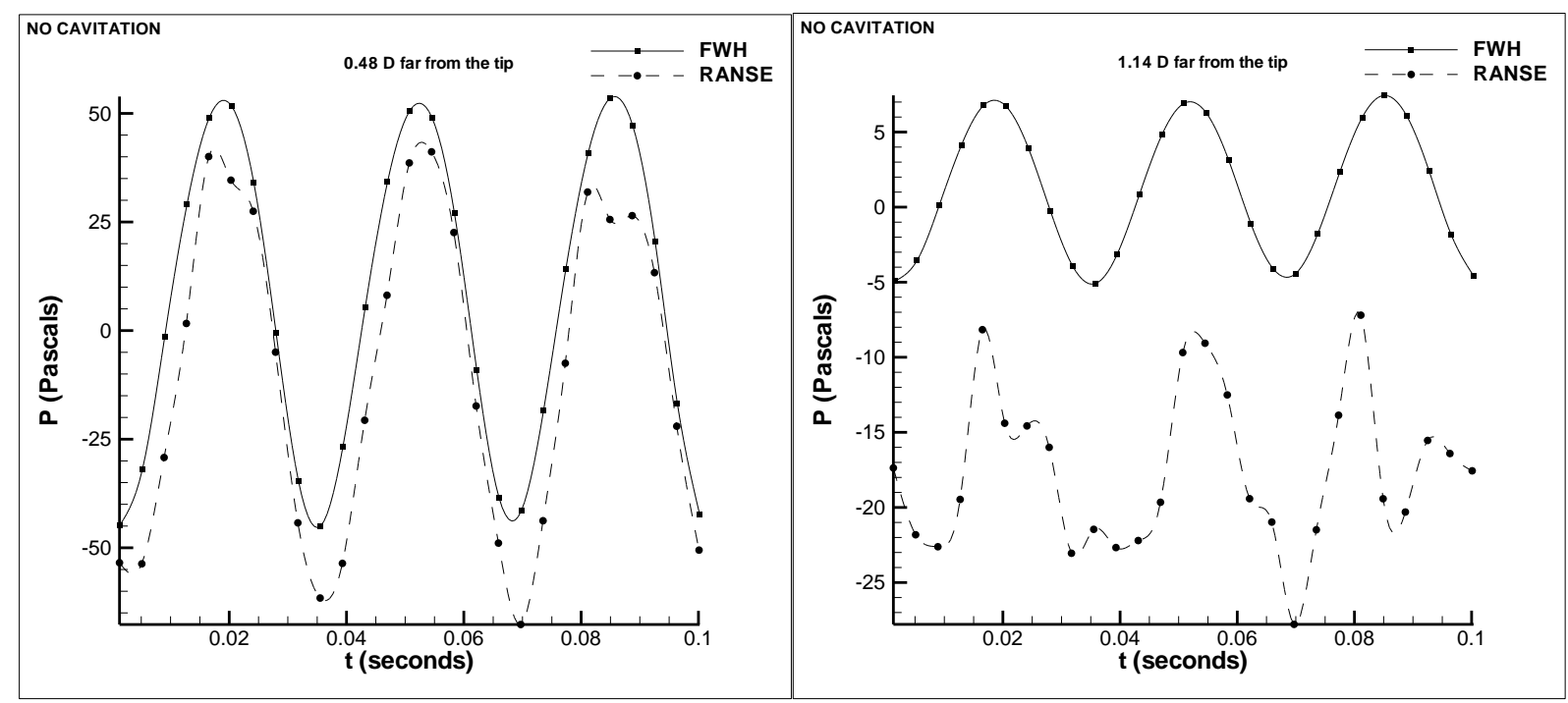

Figure 8. Pressure fluctuations induced by one propeller rotation at hydrophones 3 (left) and 4 (right) for non-cavitating case.

Sound pressure levels in the frequency domain are obtained by Fast Fourier Transform (FFT) using the pressure data in time domain provided in Figure 7 and Figure 8 . This study only focuses on the tonal noise and frequencies up to $200 \mathrm{~Hz}$ were given. Urick (1983) states that a frequency range of $0 \leq f \leq$ $500 \mathrm{~Hz}$ is sufficient to assess hydroacoustic performance of ships as this range covers the most dominant part of ship noise. Frequencies over $200 \mathrm{~Hz}$ do not exhibit new features to be explained in this study, therefore, they are not given for presenting clearer figures. Numerical hydroacoustic measurements are given in Figure 9 for hydrophones 1 and 2 and Figure 10 for hydrophones 3 and 4 . Sound pressure levels in these figures were calculated by;

$$
S P L=20 * \log \left(\frac{p}{p_{\text {ref }}}\right)
$$

where $p_{\text {ref }}$ denotes reference sound pressure in water in this study $\left(p_{r e f}=10^{-6} \mathrm{~Pa}\right)$.

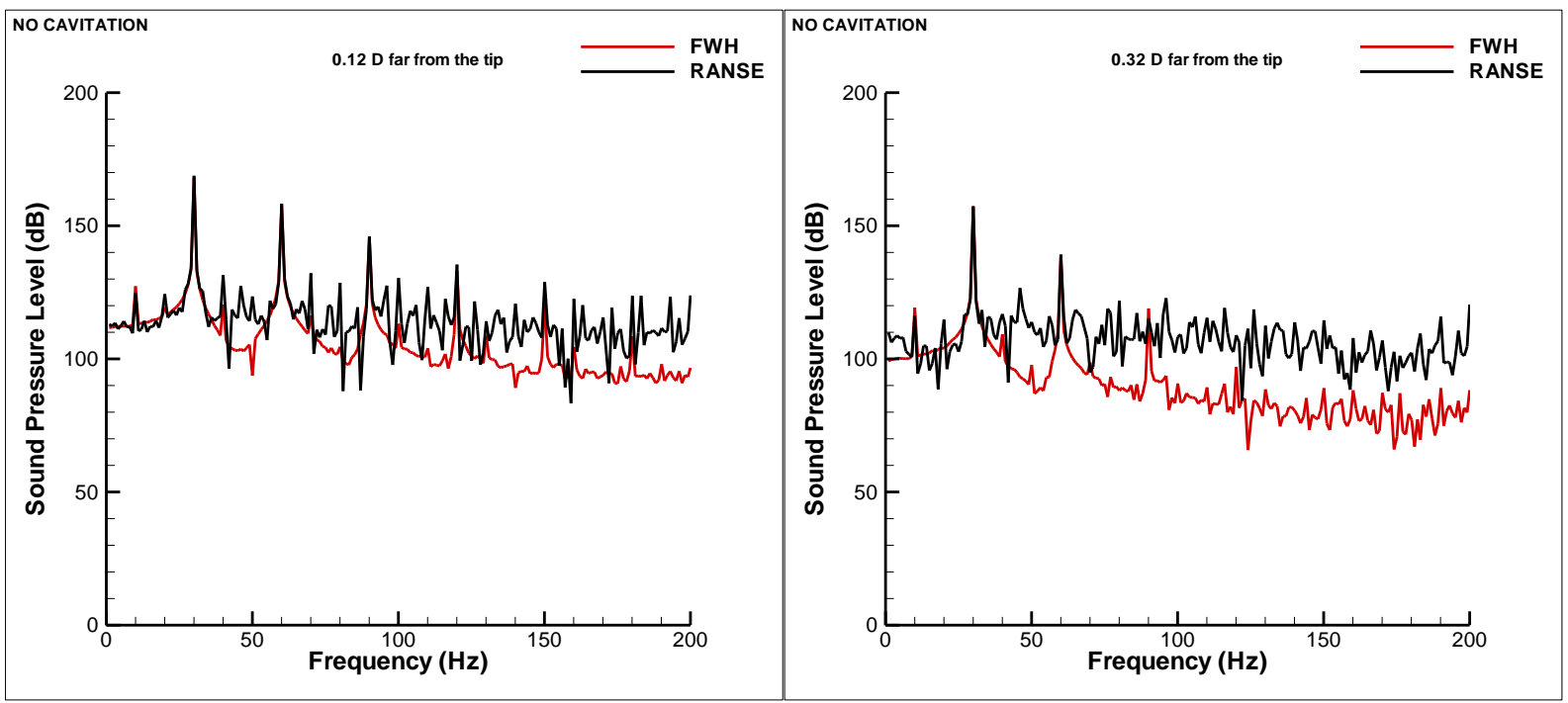

Figure 9. Sound pressure levels induced by the propeller at hydrophones 1 (left) and 2 (right) for noncavitating case. 


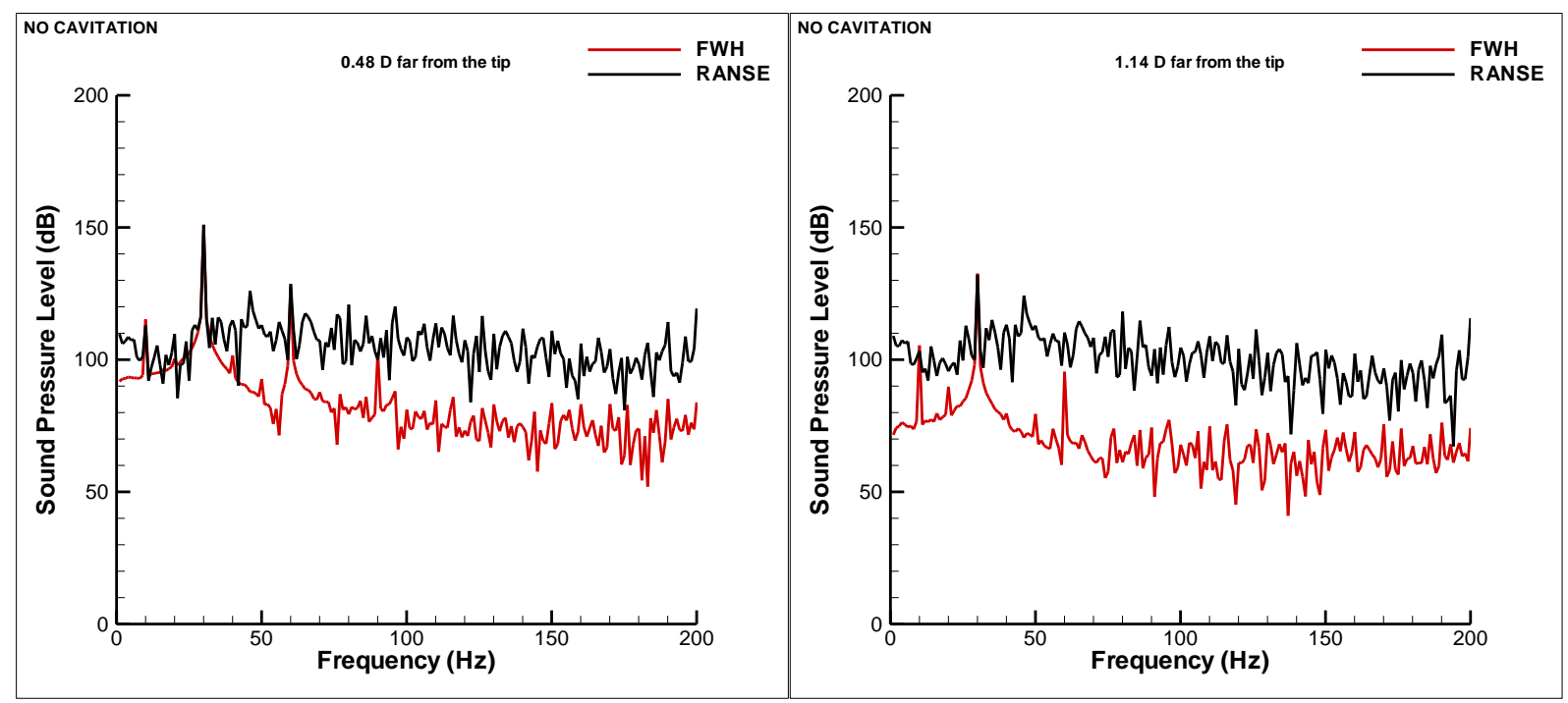

Figure 10. Sound pressure levels induced by the propeller at hydrophones 3 (left) and 4 (right) for non-cavitating case.

Coherent pressure fluctuations for hydrophones 1 and 2 are also reflected to sound pressure levels as expected. It can be stated that locations of hydrophones 1 and 2 are "perfect" for RANSE as they are in the near-field and in a position where vorticity plays almost no role. This can also be seen from Figure 11 that vorticity is very low / not present in the propeller plane. Additional refinements in the wake covering the possible tip vortex region of the propeller are advised by a recent study in the literature (Yilmaz et al., 2019). However; propeller swirl, as given in Figure 11, is not effective for the receivers included in this study.

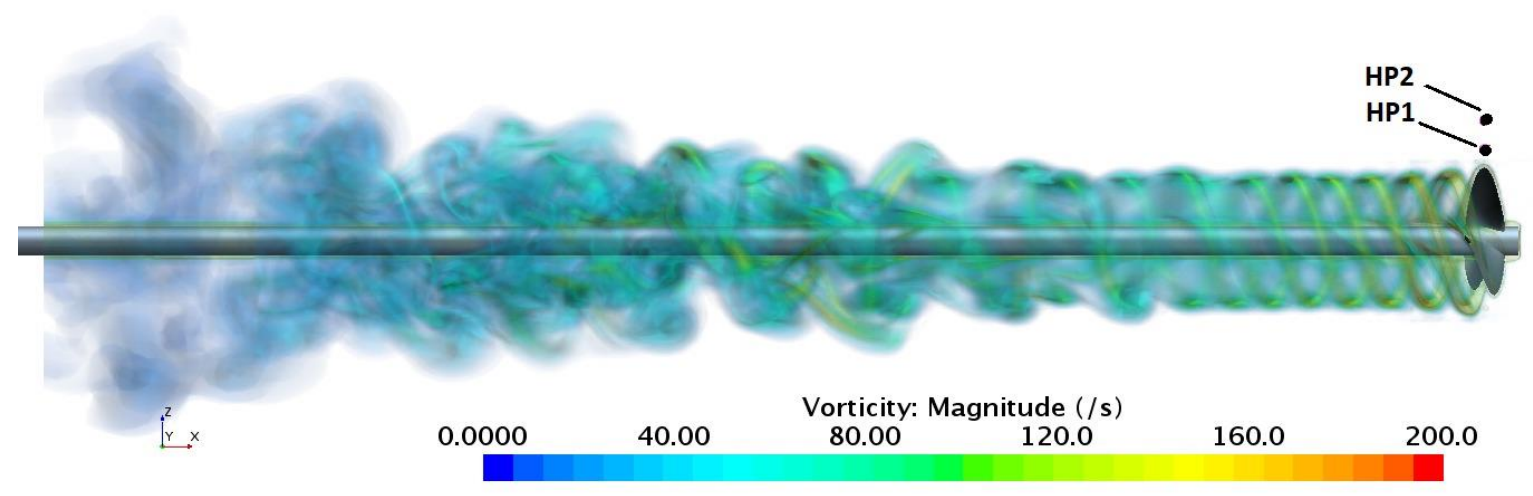

Figure 11. Vorticity magnitude in the wake and the propeller plane of DTMB 4119.

RANSE was evaluated from sound pressure levels in the frequency domain by comparing results with FWH at sub-harmonic, fundamental and harmonic frequencies of the propeller. Peaks at subharmonics frequencies of $10 \mathrm{~Hz}$ and $20 \mathrm{~Hz}$ are clearly visible for hydrophone 1 and noticeable for hydrophones 2 and 3 . However, these are not visible in hydrophone 4 . Similar comments can also be made for harmonic frequencies: these are clearly visible for hydrophone 1 and unclear for hydrophone 4. On the other hand, RANSE was excellent in capturing the peak at the fundamental frequency of $30 \mathrm{~Hz}$ regardless of the distance. Digital values for hydrophones 1 and 4 obtained by both methods are given in Table 5 for the harmonics of the blade passage frequency. It may be seen from this table that compressibility results in reduction of SPL in almost all the frequency spectrum covered. Sound moves in elastic media and are subject to losses. Pressure signals are delayed when compressibility is 
introduced in the flow. This results in reduction of SPL as the sound moves away from the sound source.

Table 5. Harmonics of the propeller at hydrophones 1 and 4 for non-cavitating case.

\begin{tabular}{|c|c|c|c|c|}
\cline { 2 - 5 } \multicolumn{1}{c|}{} & \multicolumn{2}{c|}{ Hydrophone \#1 } & \multicolumn{2}{c|}{ Hydrophone \#4 } \\
\hline $\begin{array}{c}\text { Frequency } \\
(\mathrm{Hz})\end{array}$ & FWH (dB) & $\begin{array}{c}\text { Incompressible } \\
\text { Solver }(\mathrm{dB})\end{array}$ & FWH (dB) & $\begin{array}{c}\text { Incompressible } \\
\text { Solver (dB) }\end{array}$ \\
\hline 30 & 167.9 & 168.8 & 132.6 & 132.1 \\
\hline 60 & 158.0 & 158.3 & 95.4 & 110.3 \\
\hline 90 & 146.0 & 145.9 & 74.3 & 93.9 \\
\hline 120 & 133.1 & 135.4 & 61.0 & 104.1 \\
\hline 150 & 120.3 & 128.9 & 73.5 & 103.8 \\
\hline 180 & 108.4 & 123.7 & 67.4 & 95.0 \\
\hline 210 & 99.8 & 127.6 & 76.5 & 108.6 \\
\hline 240 & 99.8 & 108.3 & 71.9 & 114.2 \\
\hline
\end{tabular}

With respect to the frequency distributions provided in Figures 9 and 10, overall sound pressure levels (OASPL) are calculated. In this study, OASPL is calculated by;

$$
O A S P L=10 * \log _{10}\left(\int_{0}^{f_{\max }} 10^{\frac{S P L(f)}{10}} \cdot d f\right)
$$

where $f_{\max }=263 \mathrm{~Hz}$ (due to Nyquist theorem where $\Delta t \cong 0.0019$ in computations) and $S P L(f)$ is the sound pressure level in $\mathrm{dB}$ at each frequency in the spectrum. OASPL values at hydrophones 1 and 4 are given in Table 6 for both methods. RANSE results of hydrophone 4 were not depicted in this table due to inconvenient pressure distributions given in Figure 8 (right).

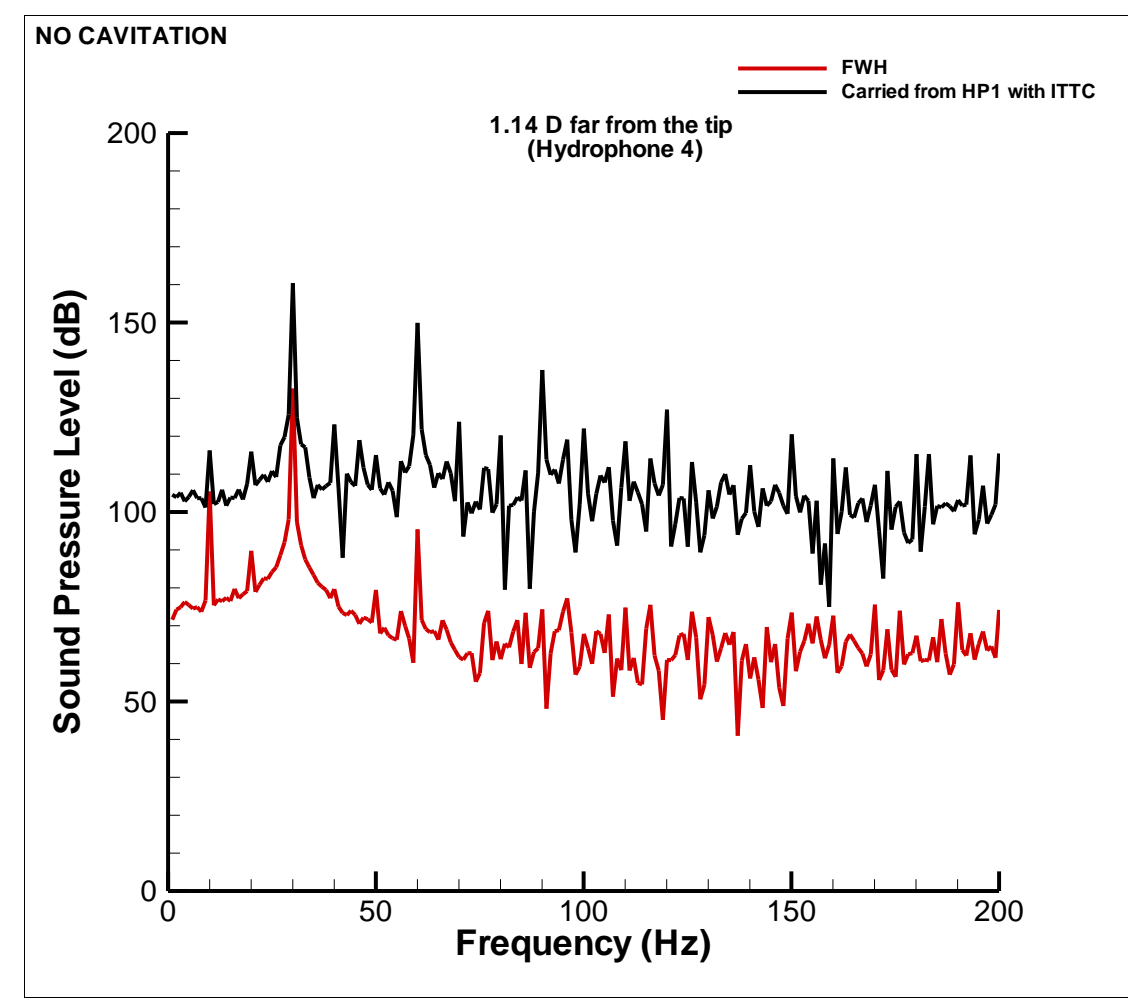

Figure 12. Sound pressure levels calculated at hydrophone 4 from hydrophone 1 with ITTC distance normalization for non-cavitating case. 
Hydrodynamic solvers are sufficient to assess the near-field noise but start to become insufficient as the hydrophone distance increases. ITTC recommends a formula called "distance normalization" equation to compute noise levels at desired locations in the flow. This equation is given as (ITTC, 7.502-01-05)

$$
S P L_{\text {ITTC }}=S P L+20 * \log _{10}\left[\frac{d}{d_{\text {ref }}}\right]
$$

In this equation; $S P L_{\text {ITTC }}$ is the sound pressure level in decibels corrected by the distance normalization equation, $S P L$ is the measured sound pressure level at the hydrophone, $d$ is the distance of the hydrophone from the noise source and $d_{\text {ref }}$ is the reference distance. This equation is actually given for experimental facilities to transfer a measured value from a hydrophone to a reference distance of $d_{r e f}=1 \mathrm{~m}$.

Distance normalization equation in this study is used to transfer near-field results obtained from the incompressible solver to the far-field without solving the FWH equation. The equation was applied both to OASPL value (Table 6) and SPL in the frequency domain (Figure 12). Distance normalization equation generally returned a higher result as compared to the actual FWH solution. ITTC distance normalization equation actually relies on inverse distance law (inverse-square law in some texts) and is valid when a point noise source radiates sound evenly. Since a marine propeller is a volume consisting of many points and there is an inflow which disturbs even sound directivity, ITTC distance normalization equation can only provide a loose prediction of sound in the far-field; as given in Table 6 and Figure 12.

Table 6. Overall sound pressure levels at hydrophones 1 and 4 for non-cavitating case.

\begin{tabular}{|c|c|c|c|}
\hline & HP \#1 & HP \#4 & $\begin{array}{c}\text { HP \#4 } \\
\text { (from HP \#1 with ITTC) }\end{array}$ \\
\hline Incompressible solver & 169.2 & - & 160.8 \\
\hline FWH & 168.3 & 132.6 & - \\
\hline
\end{tabular}

\subsection{Cavitating case}

In the cavitating propeller case, hydrophone locations were similar so that direct comparisons with non-cavitating cases can be made. Due to limitations of RANSE, focus of this study was on sheet cavitation. The effectiveness of RANSE approach in modelling the sheet cavitation on the propeller was mentioned by (Gaggero et al., 2014) in their study. Bursting of vapor bubbles, which significantly increase noise levels for very short durations, was left out of the problem; as this would require additional models to account for the collapse of the cavity. Cavitating case for DTMB 4119 propeller was numerically simulated by using the simulation conditions of the non-cavitating case; except the atmospheric pressure. Cavitation was achieved by setting the atmospheric pressure to a lower value and decreasing the cavitation number given in equation (15). Bagheri et al. (2017) have also followed such a strategy to obtain a cavitating propeller case, where they have examined the inception and development of sheet cavitation to understand the effects of rotational speed on propeller radiated noise. 


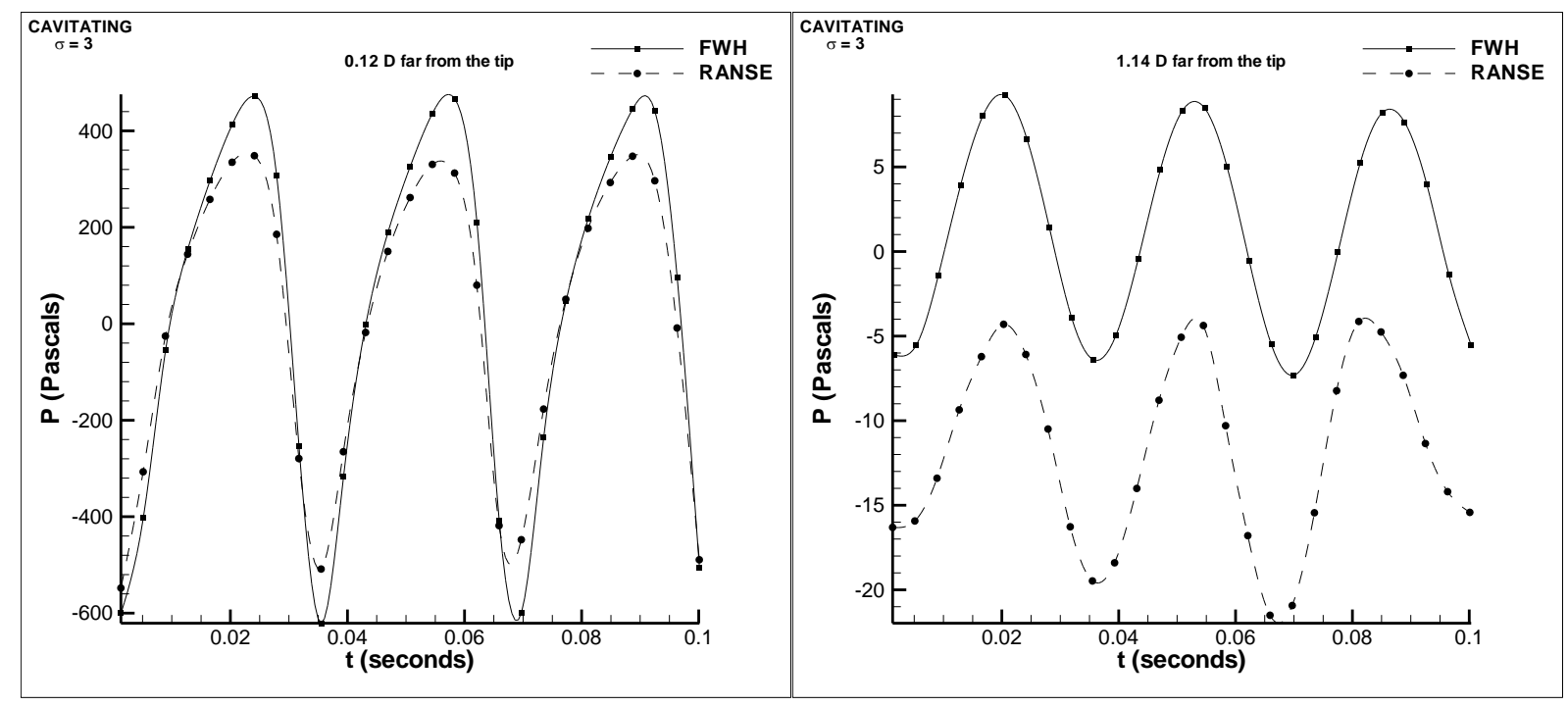

Figure 13. Pressure fluctuations induced by one propeller rotation at hydrophones 1 (left) and 4 (right) for cavitating case. $\sigma=3$.

Table 7. Simulation conditions and geometric properties of the cavitating DTMB 4119 propeller.

\begin{tabular}{|c|c|c|c|c|c|c|c|}
\hline $\mathrm{D}(\mathrm{m})$ & $\mathrm{n}(\mathrm{rps})$ & $\mathrm{V}(\mathrm{m} / \mathrm{s})$ & $\mathrm{P}_{0}(\mathrm{~Pa})$ & $\mathrm{J}(-)$ & $\sigma(-)$ & $\mathrm{Ma}_{\text {tip }}(-)$ & $1^{\text {st }} \mathrm{BPF}(\mathrm{Hz})$ \\
\hline 0.3048 & 10 & 1.524 & 16240 & 0.5 & 3 & $6.4^{*} 10^{-3}$ & 30 \\
\hline
\end{tabular}

Pressures obtained from numerical simulations for the cavitating case are presented in Figure 13 for hydrophones 1 and 4. It was observed that the results of the incompressible solver are somewhat in accordance with FWH in the near-field but it completely fails in the far-field. When the results given in Figure 13 are compared with the results given in Figure 7 (left) and Figure 8 (right), it can be seen that pressures obtained from the incompressible solver are nearly irresponsive of cavitation number. However, it is known that cavitation is a physical phenomenon which increases noise dramatically. Such increase in noise with cavitation should be reflected as an increase in pressure. Incompressible solver was unsatisfactory to represent this increase while the implementation of FWH clearly reflected this increase.

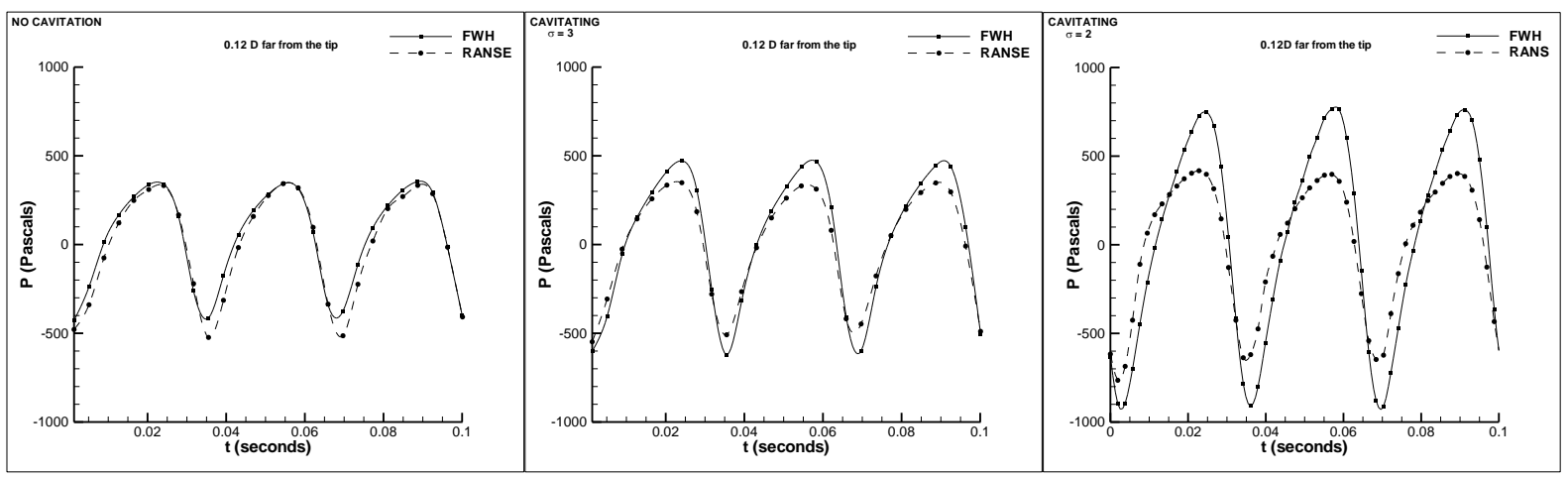

Figure 14. Effect of cavitation on acoustic pressure in the near-field (hydrophone 1). $\sigma=31.92$ (left), $\sigma=3$ (middle) and $\sigma=2$ (right).

To better understand the effect of cavitation on both methods, another numerical simulation was carried out using the same conditions given in Table 7. This time, cavitation number was selected as $\sigma=2$ by setting the static pressure to $P_{0}=11606 \mathrm{~Pa}$. Acoustic pressures obtained for hydrophone 1 
are presented in Figure 14. Changes in pressure obtained by RANSE are at a negligible level while FWH results show a major difference in pressure with decreasing cavitation number. Clearly, we cannot use RANSE results and state that sheet cavitation nearly has "no effect" on noise. Effect of cavitation on SPL was experimentally shown by (Aktas et al., 2018) recently and they note that sheet cavitation increases sound emanating from propellers especially at the $1^{\text {st }}$ BPF. Therefore, results given in Figure 15 actually point to an important deficiency of RANSE. Figure 15 exhibits pressure distributions on the propeller suction side for different cavitation numbers. There is a noticeable difference in pressures in this figure but this difference in pressure does not seem to be reflected to pressures away from the propeller surface. $\mathrm{FWH}$, on the other hand, seems to be successful in transporting surface pressures to the hydrophone location. Cavitation patterns obtained at both cavitating conditions ( $\sigma=2$ and $\sigma=$ 3) are given in Figure 16.

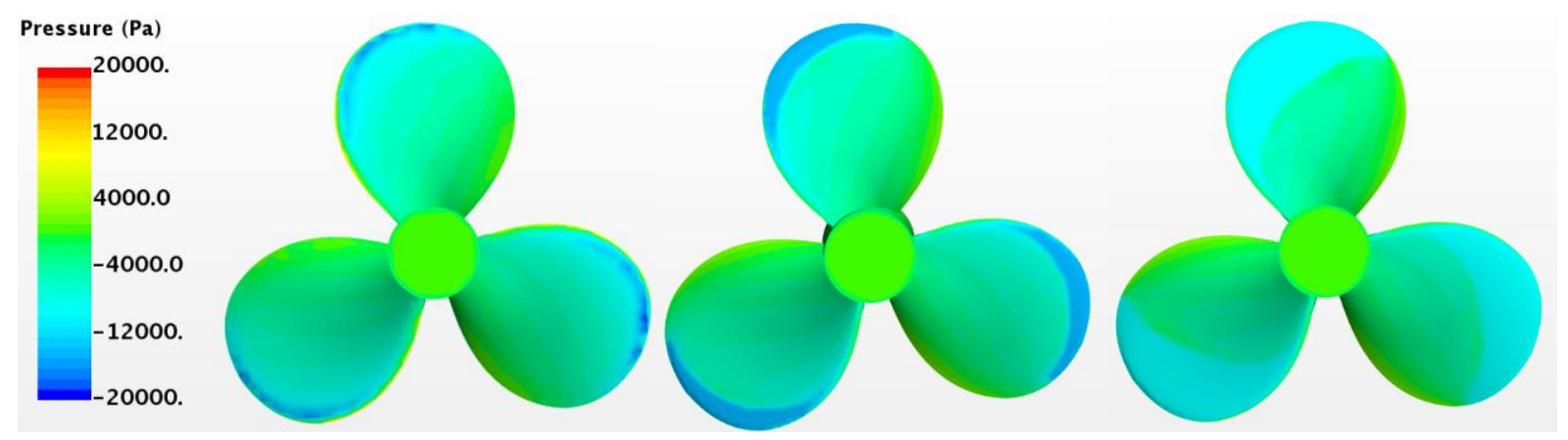

Figure 15. Pressure distributions on the propeller surface (suction side) for different cavitation numbers. $\sigma=31.92$ (left), $\sigma=3$ (middle) and $\sigma=2$ (right).

Sound pressure levels obtained by both methods for hydrophones 1 and 4 are given in Figure 17 . RANSE seems to show a remarkable accordance with FWH in the near-field (hydrophone 1). Sound pressure levels at the first blade passage frequency are given in Table 8. RANSE was able to show good agreement with $\mathrm{FWH}$ at the first blade passage frequency of the propeller. Sub-harmonic, fundamental and harmonic frequencies of the signal also seem to be coherent. However, it was shown in Figure 13 (left) that there was a noticeable difference in pressures obtained by RANSE and FWH. This difference in pressures disappears when logarithm of the pressure is taken to calculate the sound pressure level by using equation (23). On the other hand, results in the far-field were highly different. There are large deviations between RANSE and FWH in every aspect for hydrophone 4. Sub-harmonic and harmonic frequencies are not clear in RANSE and the sound pressure levels are not in a good agreement. It should also be noted here that despite generating higher pressure (Figure 13), FWH results seem to be equal or lower at each frequency in the frequency spectrum. This is correct except the fundamental (first blade passage) frequency as given in Table 8 . High pressure fluctuations lead to higher noise at the $1^{\text {st }}$ BPF.

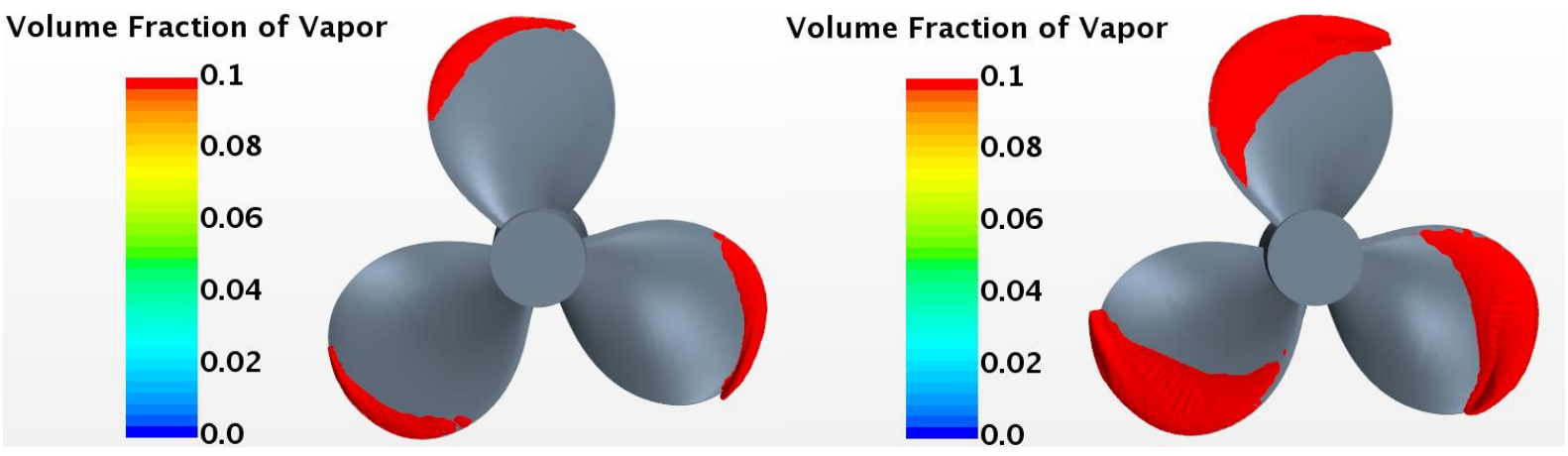

Figure 16. Cavitation pattern of the propeller. $\sigma=3$ (left), $\sigma=2$ (right). 


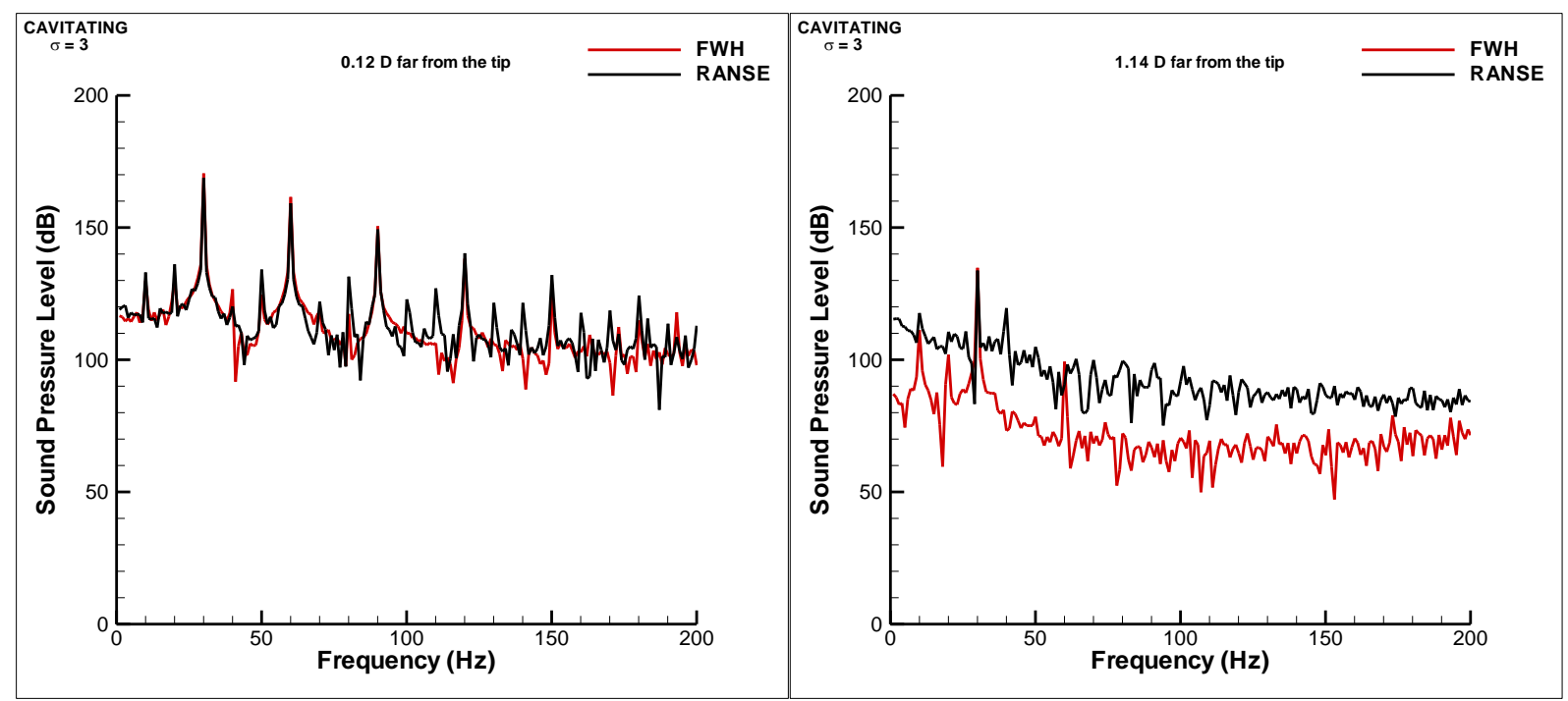

Figure 17. Sound pressure levels induced by the propeller at hydrophones 1 (left) and 4 (right) for cavitating case. $\sigma=3$.

As previously said, noise can be predicted both by direct approach (Direct Numerical Simulation, shortly DNS) and hybrid methods (incompressible + hydroacoustic solvers) in numerical simulations. However; since the sound speed is finite and sound is defined as a pressure fluctuation propagating in a medium, DNS involves compressible flow for noise prediction and offers highly accurate results. On the other hand, the hybrid method based on incompressible flow theory requires incompressibility assumption $(d \rho=0)$. According to isentropic flow hypothesis $\left(c_{0}{ }^{2}=d p / d \rho\right)$, sound speed becomes infinite in this case and pressures gathered from all possible noise sources at a fixed receiver point overlap randomly. Therefore, contribution of different noise sources at the receiver location at the right time can only be made by adding compressibility delay. This is enabled by coupling the incompressible solver by the acoustic solver. In normal operating conditions, the propeller rotational speed is low as compared to sound speed in water and due to this reason, incompressible solver might be sufficient due to negligible time shift. In this case the propeller rotational speed and loading on the blades are low which makes it possible to compare RANSE with FWH. However in cavitating cases, either the rotational speed or loading on the blades are high (sometimes both) which limits utilization of RANSE to estimate the noise of a cavitating propeller.

Table 8. Sound pressure levels at the first blade passage frequency. $\sigma=3$.

\begin{tabular}{|c|c|c|}
\cline { 2 - 3 } \multicolumn{1}{c|}{} & FWH (dB) & $\begin{array}{c}\text { Incompressible } \\
\text { Solver }(\mathrm{dB})\end{array}$ \\
\hline Point \#1 & 170.5 & 168.8 \\
\hline Point \#4 & 134.8 & 133.7 \\
\hline
\end{tabular}

Table 9. Overall sound pressure levels at hydrophones 1 and 4 for cavitating case. $\sigma=3$.

\begin{tabular}{|c|c|c|c|}
\hline & HP \#1 & HP \#4 & $\begin{array}{c}\text { HP \#4 } \\
\text { (from HP \#1 with ITTC) }\end{array}$ \\
\hline $\begin{array}{c}\text { Incompressible } \\
\text { solver }\end{array}$ & 169.3 & - & 160.9 \\
\hline FWH & 171.1 & 134.8 & - \\
\hline
\end{tabular}


Overall sound pressure levels for $\sigma=3$ case are given in Table 9. Similar to non-cavitating case, incompressible solver and FWH results are in good agreement in the near-field (hydrophone 1) while the results show discrepancy in the far-field (hydrophone 4). FWH results calculate OASPL in hydrophone 4 as $134.8 \mathrm{~dB}$. However, extrapolation of RANSE based near-field results to the far-field by ITTC distance normalization equation returns an OASPL of 160.9. The difference in results is also visible in SPL versus frequency distribution given in Figure 18.

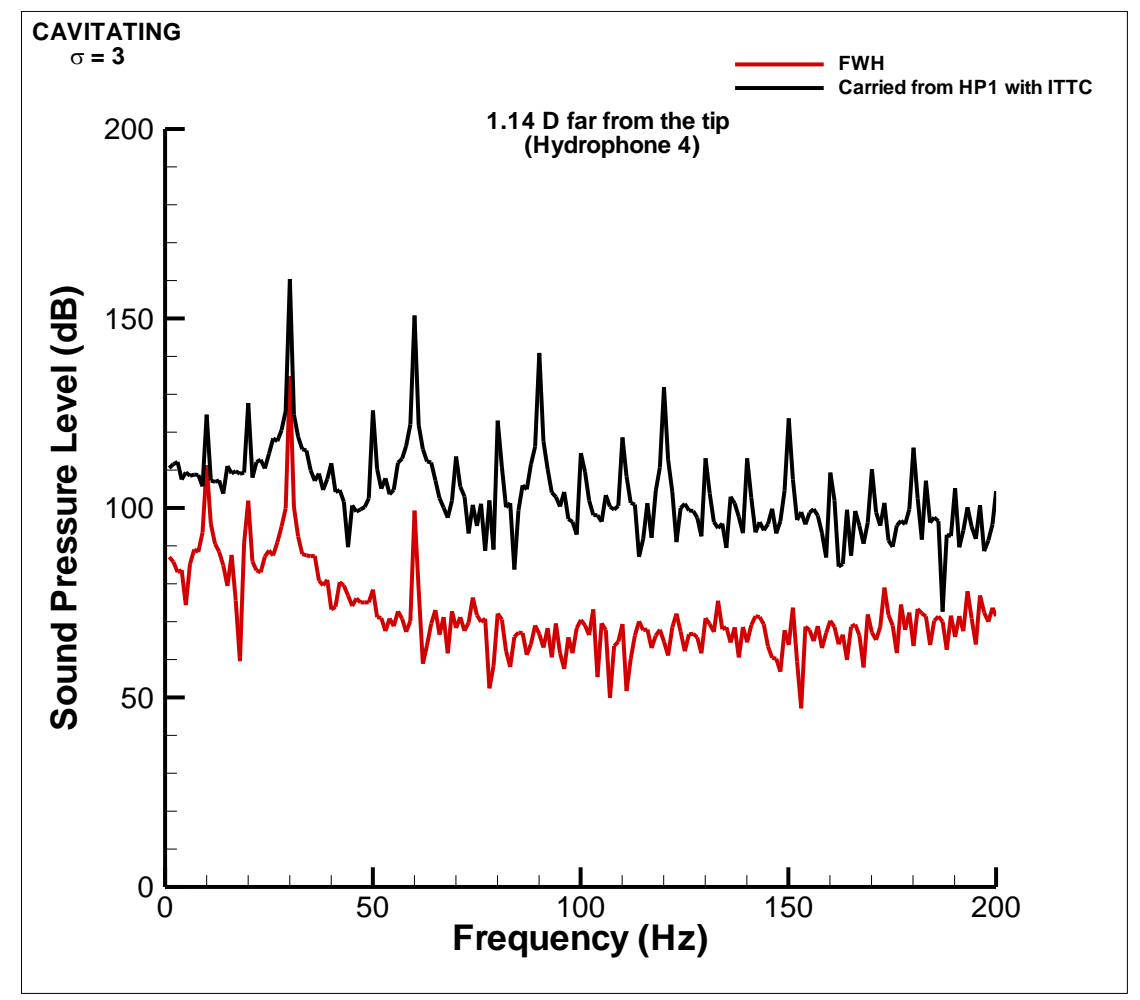

Figure 18. Sound pressure levels calculated at hydrophone 4 from hydrophone 1 with ITTC distance normalization for cavitating case. $\sigma=3$.

\section{Discussion}

There are several outcomes of this study and the first one addresses the computations. Time step size selection is a very important step in CFD computations. Rotating blades of the propeller create oscillations in pressure in the fluid domain. To correctly capture the periodic nature of these pressure fluctuations, a time step size selection formula was proposed in this study. By using this formula, oscillations in pressure could clearly be observed.

Second outcome is related to ITTC distance normalization equation. This equation is generally used to calculate sound pressure levels at a desired point in the flow by taking a reference point with previously known SPL. It is shown in this study (as well as in few others in the literature) that incompressible hydrodynamic solvers are sufficient to calculate the hydroacoustic properties of a propeller in the nearfield. Linking these two facts, it is possible to say that propeller noise can be calculated by using incompressible hydrodynamic solvers and then the results can be extended to anywhere in the flow by the ITTC distance normalization equation. With this approach, FWH equation can be excluded from calculations. This study shows that this is incorrect. 
ITTC distance normalization equation (known as the inverse distance law in acoustics) is given for an infinitely small noise source in stationary fluid. Neither Doppler effect, nor sound directivity are considered. Considering that a propeller consists of surfaces formed of many points and there is an inflow coming onto it; validity of this equation can be discussed. In this study, it was shown that extending these results to far-field by using ITTC distance normalization equation does not provide desirable results when compared with the results generated by using FWH equation. Hydroacoustic results generated by incompressible hydrodynamic solver may be satisfactory in near-field. However for far-field, implementation of FWH equation is mandatory to obtain accurate results. ITTC distance normalization equation may still be valid to carry results in nearby distances but there is no limitation of distance in the guide (ITTC, 7.5-02-01-05).

Lastly, inadequacy of the incompressible hydrodynamic solvers for cavitating cases were addressed. In the literature, it is previously shown that incompressible hydrodynamic solvers are satisfactory in resolving the noise induced by propeller in near-field. However, in this study, it is shown that this is only valid for non-cavitating cases. It is expected that presence of cavitation increases the pressure in the fluid domain; however, incompressible solvers were irresponsive of the cavitation number. In cavitating cases, only a slight but insignificant pressure increase was observed which would lead to a physically incorrect conclusion that sound pressure levels do not change with cavitation. This shows the inadequacy of incompressible hydrodynamic solvers in noise calculation for cavitating cases regardless of the location of the hydrophone.

\section{Conclusions}

In this study, effects of incompressibility assumption in hydroacoustic performance prediction of a marine propeller were investigated numerically. After explaining the grid structure and the numerical algorithms involved in simulations; a suitable time step size selection procedure was presented. Verification and validation study revealed that numerical simulation uncertainties were calculated within acceptable limits. Later, noise estimations using incompressible solver only and incompressible + hydroacoustic solver were compared.

It was found out that RANSE is able to produce satisfactory results in the near-field for the noncavitating case. However, RANSE showed lower reliability for cavitating cases even in the near-field region. For far-field, this option cannot be considered in neither cases.

After it was determined that RANSE is generating compatible results in the near-field, whether it was possible to extend near-field results to far-field by using ITTC distance normalization equation was investigated. It was found out that coupling RANSE with the equation suggested by ITTC is not generating results in accordance with the FWH equation.

Future research will be conducted on adding the nonlinear terms to FWH equation by changing the integration surface type to "porous". As it was shown in this study, RANSE was not satisfactory even in near-field zone for cavitating case. More advanced turbulence models such as DES or LES might help resolving the flow characteristics. It is considered that utilization of a more advance turbulence model with nonlinear terms will lead to a better understanding of the relationship between cavitation and sound pressure levels. 


\section{Acknowledgements}

This study was supported by the Presidency of Defence Industries of the Republic of Turkey through ITUNOVA Technology Transfer Office.

\section{References}

- Aktas, B., Atlar, M, Fitzsimmons, P., Weichao, S., 2018. An advanced joint time-frequency analysis procedure to study cavitation-induced noise by using standard series propeller data. Ocean Engineering, 170, 329-350.

- Andrew, R.K., Howe, B.M., Mercer, J.A., Dzieciuch, M.A., 2002. Ocean ambient sound: comparing the 1960s with the 1990s for a receiver off the California coast. Acoustic Research Letters Online, 3, 65-70.

- Bagheri, M.R., Seif, M.S., Mehdigholi, H., Yaakob, O., 2017. Analysis of noise behaviour for marine propellers under cavitating and non-cavitating conditions. Ships and Offshore Structures, 12(1), 1-8.

- Bertschneider, H., Bosschers, J., Choi, G.., Ciappi, E., Farabee, T., Kawakita, C., Tang, D., 2014. Final Report and Recommendations to the 27th ITTC in Specialist Committee on Hydrodynamic Noise.

- Brennen, C. E., 2008. Cloud Cavitation: The Good, The Bad and the Bubbly. WIMRC FORUM 2008, United Kingdom.

- Farassat, F. 2007. Derivation of Formulations 1 and 1A of Farassat. NASA/TM-2007-214853, Langley Research Center, Hampton, Virginia.

- Gaggero, S., Tani, G., Viviani, M., Conti, F., 2014. A Study on The Numerical Prediction of Propellers Cavitating Tip Vortex, Ocean Engineering 92, 137-161.

- Gungor, E., Ozdemir, I.B., 2018. Prediction of noise and acoustical spectrum of counterrotating propellers. Journal of Ship Research, 62(3), 166-182.

- Hallander, J., Allenstrom, B., Valdenazzi, F., Barras, C., 2012. Predicting Underwater Radiated Noise due to a Cavitating Propeller in a Ship Wake. 8th International Symposium on Cavitation (CAV2012), Singapore, pp. 1-7.

- Ianniello, S., De Bernardis, E., 2015. Farassat's formulations in marine propeller hydroacoustics. Int. J. Aeroacoustics 14, 87-103.

- Ianniello, S., Muscari, R., Di Mascio, A., 2013. Ship underwater noise assessment by the acoustic analogy. Part I: nonlinear analysis of a marine propeller in a uniform flow. J. Mar. Sci. Technol. 18, 547-570.

- Ianniello, S., Muscari, R., Mascio, A.D., 2011. Hydroacoustic characterization of a marine propeller through the acoustic analogy. Sustainable Maritime Transportation and Exploitation of Sea Resources.ISBN 978-0-415-62081-9.

- IMO, 2014. Guidelines for the reduction of underwater noise from commercial shipping to address adverse impacts on marine life.

- ITTC, 2014. 7.5-02-01-05 Model scale noise measurements, Recommended Procedures and Guidelines.

- ITTC, Specialist Committee on Hydrodynamic Noise, 2014.

- IWC, 2009. Report of the scientific committee. Journal of Cetacean Research and Management, 11 (suppl.), Section 12. Environmental Concerns, 46-47.

- Jessup, S., 1989. An experimental investigation of viscous aspects of propeller blade flow. PhD Thesis, The Catholic University of America. 
- Kellett, P., Turan, O., Incecik, A., 2013. A study of numerical ship underwater noise prediction. Ocean Eng. 66, 113-120.

- Leaper, R., Renilson, M., Ryan, C., 2014. Reducing underwater noise from large commercial ships: Current status and future directions. Journal of Ocean Technology, 9(1), 50-69.

- Lidtke, A.K., Humphrey, V.F., Turnock, S.R., 2016. Feasibility study into a computational approach for marine propeller noise and cavitation modelling. Ocean Eng. 120, 152-159.

- Lighthill, M.J., 1952. On sound generated aerodynamically I. General theory. Proc R Soc Lond A 211, 564-587.

- Lloyd, T.P., Rijpkema, D., Wijngaarden, E. van, 2015. Marine propeller acoustic modelling: comparing CFD results with an acoustic analogy method. Fourth International Symposium on Marine Propulsors, spm'15, Austin, Texas.

- Noughabi, A.K., Bayati, M., Tadjfar, M., 2017. Investigation of Cavitation Phenomena on Noise of Underwater Propeller, in: Proceedings of the ASME 2017, USA.

- Moussa, K., 2014. Computational Modeling of Propeller Noise NASA SR-7A. MSc Thesis Waterloo, Ontario, Canada

- Porcacchia, F., Gennaretti, M., Testa, C., Zaghi, S., Greco, L., Dubbioso, G., Muscari, R., 2018. Inclined-flow propeller hydroacoustics by the permeable Ffowcs Williams Hawkings equation. 25th International Congress on Sound and Vibration ICSV25, Hiroshima, Japan.

- Seol, H., Jung, B., Suh, J.C., Lee, S., 2002. Prediction of non-cavitating underwater propeller noise. Journal of Sound and Vibration, 257(1), 131-156.

- Salvatore, F., lanniello, S., 2003. Preliminary results on acoustic modelling of cavitating propellers. Comput. Mech. 32, 291-300.

- Salvatore, F., Testa, C., \& Greco, L., 2009. Coupled hydrodynamics-hydroacoustics BEM modelling of marine propellers operating in a wakefield. Proceedings of SMP, 9, 537-547.

- Seol, H., Suh, J.-C., Lee, S., 2005. Development of hybrid method for the prediction of underwater propeller noise. J. Sound Vib. 288, 345-360.

- Stern, F., Wilson, R.V., Coleman, H.W., Paterson, E.G., 2001. Comprehensive approach to verification and validation of CFD simulations - Part 1: Methodology and procedures, Journal of Fluids Engineering - Transactions of the ASME, 123(4), 793-802.

- Testa, C., lanniello, S., Salvatore, F., 2018. A Ffowcs Williams and Hawkings formulation for hydroacoustic analysis of propeller sheet cavitation. J. Sound Vib. 413, 421-441.

- Urick, R.J., 1983. The noise background of the sea: ambient noise level. Principles of Underwater Sound. 202-236.

- Viitanen, V. M., Siikonen, T. I. M. O., (2017, May). Numerical simulation of cavitating marine propeller flows. In Proceedings of the 9th National Conference on Computational Mechanics (MekIT'17), Trondheim, Norway (pp. 11-12).

- Williams, J.E.F., Hawkings, D.L., 1969. Sound generation by turbulence and surfaces in arbitrary motion. Phil Trans R Soc Lond A 264, 321-342.

- Yilmaz, N., Atlar, M., Khorasanchi, M., 2019. An improved Mesh Adaption and Refinement approach to Cavitation Simulation (MARCS) of propellers, Ocean Engineering, 171, 139-150. 